\title{
All tree-level amplitudes in massless QCD
}

\author{
Lance J. Dixon, ${ }^{a, b}$ Johannes M. Henn, ${ }^{c}$ Jan Plefka, ${ }^{c}$ and Theodor Schuster ${ }^{c}$ \\ ${ }^{a}$ Theory Group, Physics Department, CERN, \\ CH-1211 Geneva 23, Switzerland \\ ${ }^{b}$ SLAC National Accelerator Laboratory, Stanford University, \\ Stanford, CA 94309, U.S.A. \\ ${ }^{c}$ Institut für Physik, Humboldt-Universität zu Berlin, \\ Newtonstraße 15, D-12489 Berlin, Germany \\ E-mail: lance@slac.stanford.edu, henn@physik.hu-berlin.de, \\ plefka@physik.hu-berlin.de, theodor@physik.hu-berlin.de
}

ABSTRACT: We derive compact analytical formulae for all tree-level color-ordered gauge theory amplitudes involving any number of external gluons and up to four massless quarkanti-quark pairs. A general formula is presented based on the combinatorics of paths along a rooted tree and associated determinants. Explicit expressions are displayed for the next-to-maximally helicity violating (NMHV) and next-to-next-to-maximally helicity violating (NNMHV) gauge theory amplitudes. Our results are obtained by projecting the previously-found expressions for the super-amplitudes of the maximally supersymmetric super Yang-Mills theory $(\mathcal{N}=4 \mathrm{SYM})$ onto the relevant components yielding all gluongluino tree amplitudes in $\mathcal{N}=4 \mathrm{SYM}$. We show how these results carry over to the corresponding QCD amplitudes, including massless quarks of different flavors as well as a single electroweak vector boson. The public Mathematica package GGT is described, which encodes the results of this work and yields analytical formulae for all $\mathcal{N}=4 \mathrm{SYM}$ gluongluino trees. These in turn yield all QCD trees with up to four external arbitrary-flavored massless quark-anti-quark pairs.

KeYwords: QCD Phenomenology

ARXIV EPRINT: 1010.3991 


\section{Contents}

1 Introduction and conclusions 1

2 Color-ordering and spinor-helicity formalism 4

3 From $\mathcal{N}=4$ SYM to QCD tree amplitudes 5

4 All gluon tree amplitudes $\quad 12$

$5 \quad$ All single-flavor quark-anti-quark-gluon trees 15

$6 \quad$ All gluon-gluino tree amplitudes in $\mathcal{N}=4$ super Yang-Mills 18

$\begin{array}{lll}7 & \text { Proof of the master formula } & 18\end{array}$

A Explicit formulae for gluon trees $\quad 22$

A.1 NMHV amplitudes 23

$\begin{array}{lll}\text { A.2 } & \mathrm{N}^{2} \mathrm{MHV} \text { amplitudes } & 23\end{array}$

B Explicit formulae for trees with fermions $\quad 25$

B.1 MHV amplitudes $\quad 25$

B.2 NMHV amplitudes 26

B.2.1 Two fermions 26

$\begin{array}{ll}\text { B.2.2 Four fermions } & 27\end{array}$

B.2.3 Six fermions $\quad 32$

B.3 $\mathrm{N}^{2} \mathrm{MHV}$ amplitudes 33

B.3.1 Two fermions 33

$\begin{array}{lll}\text { B.3.2 Four fermions } & 34\end{array}$

B.3.3 Six fermions 34

C The Mathematica package GGT $\quad 35$

\section{Introduction and conclusions}

Scattering amplitudes play a central role in gauge theory. At a phenomenological level, they are critical to the prediction of cross sections at high-energy colliders, for processes within and beyond the Standard Model. Efficient evaluation of scattering amplitudes involving many quarks and gluons is particularly important at machines such as the Large Hadron Collider (LHC), in which complex, multi-jet final states are produced copiously and complicate the search for new physics. 
Tree amplitudes can be used to predict cross sections at leading order (LO) in the perturbative expansion in the QCD coupling $\alpha_{s}$. Such results are already available numerically for a wide variety of processes. Programs such as MAdGraph [1, 2], CompHEP [3], and AMEGIC $++[4]$ are based on fast numerical evaluation of Feynman diagrams. Other methods include the Berends-Giele (off shell) recursion relations [5], as implemented for example in COMIX [6], and the related ALPHA [7, 8] and HELAC [9, 10] algorithms based on Dyson-Schwinger equations, as well as O'MEGA/WHIZARD [11, 12]. The computation time required in these latter methods scales quite well with the number of legs.

On the formal side, the properties of scattering amplitudes have long provided numerous clues to hidden symmetries and dynamical structures in gauge theory. It was recognized early on that tree amplitudes in gauge theory are effectively supersymmetric [13, 14], so that they obey supersymmetric $S$-matrix Ward identities $[15,16]$. Soon thereafter, Parke and Taylor [17] discovered a remarkably simple formula for the maximally-helicity-violating (MHV) amplitudes for $n$-gluon scattering, which was proven by Berends and Giele [5], and soon generalized to $\mathcal{N}=4$ super Yang-Mills theory (SYM) by Nair [18].

Later, it was found that this simplicity also extends to the loop level, at least for $\mathcal{N}=4$ super Yang-Mills theory [19, 20]. These results were obtained using the unitarity method, which constructs loop amplitudes by sewing together tree amplitudes (for recent reviews see refs. [21, 22]). After Witten [23] reformulated gauge theory in terms of a topological string propagating in twistor space, there was a huge resurgence of interest in uncovering new properties of scattering amplitudes and developing new methods for their efficient computation. Among other developments, Britto, Cachazo, Feng and Witten proved a new type of recursion relation $[24,25]$ for gauge theory. In contrast to the earlier offshell recursion relations, the BCFW relation uses only on-shell lower-point amplitudes, evaluated at complex momenta. A particular solution to this recursion relation was found for an arbitary number of gluons in the split-helicity configuration $(-\cdots-+\cdots+)[26]$.

The BCFW recursion relation was then recast as a super-recursion relation for the tree amplitudes of $\mathcal{N}=4$ super Yang-Mills theory, which involves shifts of Grassmann parameters as well as momenta [27]. A related construction is given in ref. [28]. The superrecursion relation of ref. [27] was solved for arbitrary external states by Drummond and one of the present authors [29]. Tree-level super-amplitudes have a dual superconformal invariance [30,31], and the explicit solution does indeed have this symmetry [29]. It is written in terms of dual superconformal invariants, which are a straightforward generalization of those that first appeared in next-to-MHV (NMHV) super-amplitudes [30, 32]. This dual superconformal invariance of tree-level amplitudes is a hallmark of the integrability of planar $\mathcal{N}=4 \mathrm{SYM}$, as it closes with the standard superconformal symmetry into an infinite-dimensional symmetry of Yangian type [33] (a recent review is ref. [34]).

The purpose of this paper is to illustrate how these more recent formal developments can reap benefits for phenomenological applications in QCD. In particular, we will evaluate the solution in ref. [29] by carrying out the integrations over Grassmann parameters that are needed to select particular external states. In addition, we will show how to extract tree-level QCD amplitudes from the amplitudes of $\mathcal{N}=4$ super Yang-Mills theory. While this extraction is simple for pure-gluon amplitudes, and those with a single massless quark 
line, it becomes a bit more intricate for amplitudes with multiple quark lines of different flavors, because of the need to forbid the exchange of scalar particles, which are present in $\mathcal{N}=4$ super Yang-Mills theory but not in QCD.

Although, as mentioned above, there are currently many numerical programs available for computing tree amplitudes efficiently, the existence of analytic expressions may provide a yet more efficient approach in some contexts. In fact, the formulae provided in this paper have already served a practical purpose: They were used to evaluate contributions from real emission in the NLO corrections to the cross section for producing a $W$ boson in association with four jets at the LHC [35]. This process forms an important background to searches for various kinds of new physics, including supersymmetry. The real-emission corrections require evaluating nine-point tree amplitudes at a large number of different phase-space points (on the order of $10^{8}$ ), in order to get good statistical accuracy for the Monte Carlo integration over phase space.

In principle, QCD tree amplitudes can also be used to speed up the evaluation of one-loop amplitudes, when the latter are constructed from tree amplitudes using a numerical implementation of generalized unitarity. Many different generalized unitarity cuts, and hence many different tree amplitudes, are involved in the construction of a single one-loop amplitude. The tree amplitudes described here enter directly into the construction of the "cut-constructible" part [20] of one-loop amplitudes in current programs such as CutTools [36-38], Rocket [39-41] and BlackHat [42]. On the other hand, the computation-time bottleneck in these programs often comes from the so-called "rational" terms. When these terms are computed using only unitarity, it is via unitarity in $D$ dimensions [40, 41, 43-47], not four dimensions. The amplitudes presented here are four-dimensional ones, so they cannot be used directly to alleviate this bottleneck for the $D$-dimensional unitarity method. However, in the numerical implementation of loop-level on-shell recursion relations [48, 49] for the rational part in BlackHAT [42], or in the OPP method used in CutTools [36-38], there are no D-dimensional trees, so this is not an issue.

An interesting avenue for future research would be to try to generalize the results presented in this paper to QCD amplitudes containing massive quarks, or other massive colored states. Massive quark amplitudes are of interest because, for example, processes that produce top quarks in association with additional jets can form important backgrounds to new physics at the LHC. States in $\mathcal{N}=4 \mathrm{SYM}$ can be given masses through a super-Higgs mechanism. This mechanism was explored recently in the context of infrared regulation of $\mathcal{N}=4 \mathrm{SYM}$ loop amplitudes [50]. However, it should be possible to generate the appropriate tree amplitudes with massive quarks, or other massive states, from the same kind of setup, once one solves the appropriate super-BCFW recursion relations.

The remainder of this paper is organized as follows. After introducing the standard technology of color-ordered amplitudes and spinor helicity we explain the strategies of how to extract QCD tree amplitudes with massless quarks from $\mathcal{N}=4 \mathrm{SYM}$ in section 3 . We also discuss how to convert these amplitudes into trees with one electroweak vector boson. Sections 4 through 6 are devoted to stating the general analytical formulae for gluon-gluino $n$-parton amplitudes in $\mathcal{N}=4 \mathrm{SYM}$, which are proven in section 7 . In appendix A we 
provide a collection of explicit results for pure-gluon trees. Explicit formulae for trees involving up to six fermions are displayed in appendix B. Finally, appendix C is devoted to a documentation of our Mathematica package GGT which implements all of the results of this paper and yields the analytical expressions for an arbitrary flavored gluon-gluino tree amplitude in $\mathcal{N}=4 \mathrm{SYM}$. The package is included in the arXiv.org submission of this article and may also be downloaded from http://qft.physik.hu-berlin.de.

\section{Color-ordering and spinor-helicity formalism}

Tree-level gluon amplitudes in non-abelian gauge theories may be conveniently separated into a sum of terms, each composed of a simple prefactor containing the color indices, multiplied by a kinematical factor known as a partial or color-ordered amplitude. For an $n$-gluon amplitude one has

$$
\mathcal{A}_{n}^{\text {tree }}\left(\left\{p_{i}, h_{i}, a_{i}\right\}\right)=g^{n-2} \sum_{\sigma \in S_{n} / Z_{n}} \operatorname{Tr}\left(T^{a_{\sigma(1)}} \ldots T^{a_{\sigma(n)}}\right) A_{n}\left(\sigma(1)^{h_{\sigma(1)}} \ldots \sigma(n)^{h_{\sigma(n)}}\right),
$$

with the argument $i^{h_{i}}$ of the partial amplitude $A_{n}$ denoting an outgoing gluon of light-like momentum $p_{i}$ and helicity $h_{i}= \pm 1, i \in[1, n]$. The $s u\left(N_{c}\right)$ generator matrices $T^{a_{i}}$ are in the fundamental representation, and are normalized so that $\operatorname{Tr}\left(T^{a} T^{b}\right)=\delta^{a b}$.

Color-ordered amplitudes of massless particles are most compactly expressed in the spinor-helicity formalism. Here all four-momenta are written as bi-spinors via

$$
\not p^{\alpha \dot{\alpha}}=\sigma_{\mu}^{\alpha \dot{\alpha}} p^{\mu},
$$

where we take $\sigma^{\mu}=(\mathbf{1}, \vec{\sigma})$ with $\vec{\sigma}$ being the $2 \times 2$ Pauli spin matrices. Light-like vectors are then expressed via the product of two spinors

$$
\not p^{\alpha \dot{\alpha}}=\lambda^{\alpha} \tilde{\lambda}^{\dot{\alpha}}
$$

For real momenta with Lorentz signature we have $\tilde{\lambda}= \pm \lambda^{*}$, with the sign being determined by the energy component of $p$. For complex momenta the spinors $\lambda$ and $\tilde{\lambda}$ are independent. Our convention is such that all gluons are outgoing. Then in eq. (2.1) each color-ordered leg is specified by a choice of spinors $\lambda_{i}$ and $\tilde{\lambda}_{i}$ along with a helicity $h_{i}= \pm 1$. Given this data the associated polarization vectors may be reconstructed from the expressions

$$
\epsilon_{+, i}^{\alpha \dot{\alpha}}=\frac{\tilde{\lambda}_{i}^{\dot{\alpha}} \mu_{i}^{\alpha}}{\left\langle\lambda_{i} \mu_{i}\right\rangle}, \quad \epsilon_{-, i}^{\alpha \dot{\alpha}}=\frac{\lambda_{i}^{\alpha} \tilde{\mu}_{i}^{\dot{\alpha}}}{\left[\lambda_{i} \mu_{i}\right]}
$$

where $\mu_{i}^{\alpha} \tilde{\mu}_{i}^{\dot{\alpha}}$ are auxiliary momenta and we use the standard notation $\langle\lambda \mu\rangle=\epsilon_{\alpha \beta} \lambda^{\alpha} \mu^{\beta}$ and $[\lambda \mu]=\epsilon_{\dot{\alpha} \dot{\beta}} \lambda^{\dot{\alpha}} \mu^{\dot{\beta}}$. Moreover we shall often use the abbreviated forms $\langle i j\rangle=\left\langle\lambda_{i} \lambda_{j}\right\rangle$ and $[i j]=\left[\tilde{\lambda}_{i} \tilde{\lambda}_{j}\right]$ in the sequel. As an essential building block of the general tree-level scattering formula we introduce the dual coordinates or region momenta $x_{i j}^{\alpha \dot{\alpha}}$ via

$$
x_{i j}^{\alpha \dot{\alpha}}:=\left(p_{i}+p_{i+1}+\cdots+\not p_{j-1}\right)^{\alpha \dot{\alpha}}=\sum_{k=i}^{j-1} \lambda_{k}^{\alpha} \tilde{\lambda}_{k}^{\dot{\alpha}}, \quad i<j
$$


$x_{i i}=0$, and $x_{i j}=-x_{j i}$ for $i>j$. We then define the scalar quantities

$$
\left\langle n a_{1} a_{2} \ldots a_{k} \mid a\right\rangle:=\left\langle n\left|x_{n a_{1}} x_{a_{1} a_{2}} \ldots x_{a_{k-1} a_{k}}\right| a\right\rangle,
$$

which we will use frequently in the following. In fact all amplitudes can be expressed in terms of the quantities $\left\langle n a_{1} a_{2} \ldots a_{k} \mid a\right\rangle$ and the spinor products $\langle i j\rangle$.

As an example of the notation and in order to give a flavor of the kinds of results we obtain, we present a compact formula for the $n$-point NMHV pure gluon amplitude in QCD

$$
\begin{aligned}
& A_{n}^{\mathrm{NMHV}}\left(i_{1}, i_{2}, n\right)=\frac{\delta^{(4)}(p)}{\langle 12\rangle \ldots\langle n 1\rangle} \times \\
& \quad\left[\sum_{i_{1}<s \leq i_{2}<t \leq n-1} \tilde{R}_{n ; s t}\left(\left\langle n i_{1}\right\rangle\left\langle n t s \mid i_{2}\right\rangle\right)^{4}+\sum_{i_{1}<s<t \leq i_{2}} \tilde{R}_{n ; s t}\left(\left\langle i_{2} n\right\rangle\left\langle n i_{1}\right\rangle x_{s t}^{2}\right)^{4}\right. \\
& \left.\quad+\sum_{2 \leq s \leq i_{1}<i_{2}<t \leq n-1} \tilde{R}_{n ; s t}\left(\left\langle i_{2} i_{1}\right\rangle\langle n t s \mid n\rangle\right)^{4}+\sum_{2 \leq s \leq i_{1}<t \leq i_{2}} \tilde{R}_{n ; s t}\left(\left\langle n i_{2}\right\rangle\left\langle n s t \mid i_{1}\right\rangle\right)^{4}\right] .
\end{aligned}
$$

Here $i_{1}, i_{2}$ and $n$ correspond to the positions of the three negative-helicity gluons. (Using cyclic symmetry, we have put one of them at position $n$ without loss of generality.) The quantities $\tilde{R}_{n ; s t}$ are simply given by

$$
\tilde{R}_{n ; s t}:=\frac{1}{x_{s t}^{2}} \frac{\langle s(s-1)\rangle}{\langle n t s \mid s\rangle\langle n t s \mid s-1\rangle} \frac{\langle t(t-1)\rangle}{\langle n s t \mid t\rangle\langle n s t \mid t-1\rangle} .
$$

with $\tilde{R}_{n ; s t}:=0$ for $t=s+1$ or $s=t+1$. Note that the above formula is given for an arbitrary number of gluons $n$. In realistic cases this number is usually small, say of the order of 9 , in which case relatively few terms are produced by the nested sums in eq. (2.7).

\section{From $\mathcal{N}=4$ SYM to QCD tree amplitudes}

In this section we discuss how to assign quantum numbers for external states in $\mathcal{N}=$ $4 \mathrm{SYM}$ in order to generate tree amplitudes for QCD with massless quarks. We then discuss the generation of tree amplitudes including an electroweak vector boson $(W, Z$ or virtual photon). From the point of view of tree amplitudes, there are two principal differences between $\mathcal{N}=4 \mathrm{SYM}$ and massless QCD. First of all, the fermions in $\mathcal{N}=4$ SYM, the gluinos, are in the adjoint representation of $s u\left(N_{c}\right)$, rather than the fundamental representation, and come in four flavors. Secondly, the $\mathcal{N}=4 \mathrm{SYM}$ theory contains six massless scalars in the adjoint representation.

Because we use color-ordered amplitudes, as discussed in section 2, the first difference is fairly unimportant. Quark amplitudes can be assembled from the same color-ordered amplitudes as gluino amplitudes, weighted with different color factors. For example, the color decomposition for amplitudes with a single quark-anti-quark pair, and the remaining $(n-2)$ partons gluons is,

$$
\mathcal{A}_{n}^{\text {tree }}\left(1_{\bar{q}}, 2_{q}, 3, \ldots, n\right)=g^{n-2} \sum_{\sigma \in S_{n-2}}\left(T^{a_{\sigma(3)}} \ldots T^{a_{\sigma(n)}}\right)_{i_{2}}^{\bar{\imath}_{1}} A_{n}^{\text {tree }}\left(1_{\bar{q}}, 2_{q}, \sigma(3), \ldots, \sigma(n)\right) .
$$


The color-ordered amplitudes appearing in eq. (3.1) are just the subset of two-gluino$(n-2)$-gluon amplitudes in which the two gluinos are adjacent.

Amplitudes with more quark-anti-quark pairs have a somewhat more intricate color structure involving multiple strings of $T^{a}$ matrices, as explained in ref. [51]. In this case, some of the color factors also include explicit factors of $1 / N_{c}$, as required to project out the $s u\left(N_{c}\right)$-singlet state for gluon exchange between two different quark lines. However, all of the required kinematical coefficients can still be constructed from suitable linear combinations of the color-ordered amplitudes for $2 k$ external gluinos and $(n-2 k)$ external gluons.

The main goal of this section will be to illustrate how to choose the flavors of the external gluinos in order to accomplish two things: (1) avoid the internal exchange of massless scalars, and (2) allow all fermion lines present to be for distinct flavors. (In some cases one may want amplitudes with (partially) identical fermions; these can always be constructed from the distinct-flavor case by summing over the relevant exchange-terms, although it may be more efficient to compute the identical-fermion case directly.) We will accomplish this goal for amplitudes containing up to four separate fermion lines, that is, eight external fermion states.

In gauge theory, tree amplitudes that contain only external gluons are independent of the matter states in the theory [13, 14]; hence they are identical between $\mathcal{N}=4 \mathrm{SYM}$ and QCD. The reason is simply that the vertices that couple gluons to the other states in the theory always produce the fermions and scalars in pairs. There are no vertices that can destroy all the fermions and scalars, once they have been produced. If a fermion or scalar were produced at any point in a tree diagram, it would have to emerge from the diagram, which would no longer have only external gluons. In other words, the pure-gluon theory forms a closed subsector of $\mathcal{N}=4 \mathrm{SYM}$.

Another closed subsector of $\mathcal{N}=4 \mathrm{SYM}$ is $\mathcal{N}=1 \mathrm{SYM}$, which contains a gluon and a single gluino. Let $g$ denote the gluon, $\tilde{g}_{A}, A=1,2,3,4$, denote the four gluinos, and $\phi_{A B}=-\phi_{B A}$ denote the six real scalars of $\mathcal{N}=4 \mathrm{SYM}$. Then the $\mathcal{N}=1 \mathrm{SYM}$ subsector is formed by $\left(g, \tilde{g}_{1}\right)$. The reason it is closed is similar to the pure-gluon case just discussed: There are vertices that produce states other than $\left(g, \tilde{g}_{1}\right)$, but they always do so in pairs. For example, the Yukawa coupling $\phi^{A B} \tilde{g}_{A} \tilde{g}_{B}, A \neq B$, can convert $\tilde{g}_{1}$ into a scalar and a gluino each carrying an index $B \neq 1$. However, this index cannot be destroyed by further interactions.

The fact that $\mathcal{N}=1 \mathrm{SYM}$ forms a closed subsector of $\mathcal{N}=4 \mathrm{SYM}$, in addition to color ordering, immediately implies that any color-ordered QCD tree amplitude for gluons, plus arbitrarily many quarks of a single flavor, is given directly by the corresponding amplitude (with $\tilde{g}_{1}$ replacing the single quark flavor) evaluated in $\mathcal{N}=4 \mathrm{SYM}$. The less trivial QCD amplitudes to extract are those for multiple fermion flavors, primarily because of the potential for intermediate scalar exchange induced by the Yukawa coupling $\phi^{A B} \tilde{g}_{A} \tilde{g}_{B}$. Figure 1 illustrates scalar exchange in an amplitude with four fermions belonging to two different flavor lines, $A \neq B$.

The key to avoiding such unwanted scalar exchange is provided by figure 2, which shows four types of vertices that could potentially couple fermion pairs to scalars and gluons. However, all four types of vertices vanish. (Recall that helicities are labeled in an 

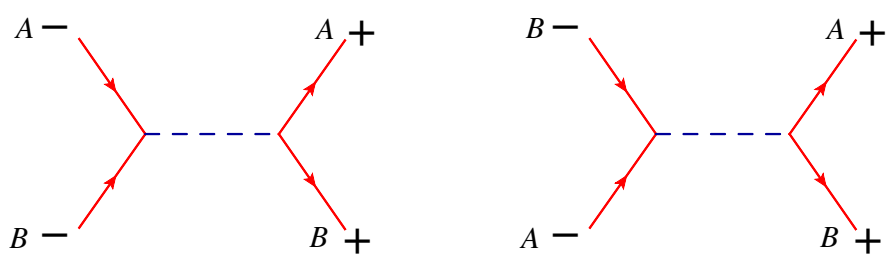

Figure 1. Unwanted scalar exchange between fermions of different flavors, $A \neq B$.

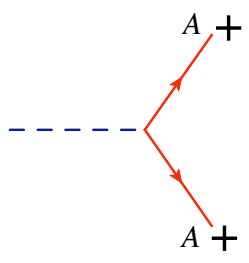

(a)

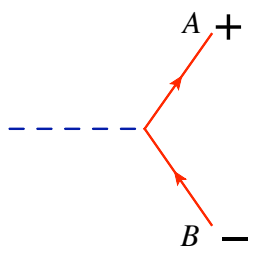

(b)

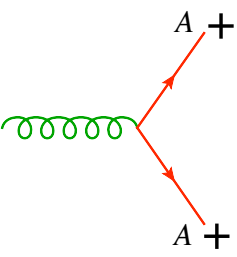

(c)

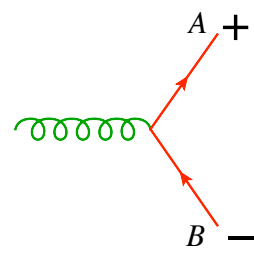

(d)

Figure 2. These vertices all vanish, as explained in the text. This fact allows us to avoid scalar exchange and control the flow of fermion flavor.

all-outgoing convention.) Case (a) vanishes because the Yukawa interaction only couples gluinos of different flavors, $A \neq B$. Cases (b) and (c) vanish because of fermion helicity conservation for the gauge interactions, and a helicity flip for the Yukawa coupling. Case (d) vanishes because gluon interactions do not change flavor.

Because the emission of gluons from fermions does not change their helicity or flavor, in analyzing whether scalar exchange can be avoided, as well as the pattern of fermion flavor flow, one can ignore the gluons altogether. For example, figure 3 shows the possible cases for amplitudes with one or two fermion lines. The left-hand side of the equality shows the desired (color-ordered) fermion-line flow and helicity assignment for a QCD tree amplitude. All gluons have been omitted, and all fermion lines on the left-hand side are assumed to have distinct flavors. The right-hand side of the equality displays a choice of gluino flavor that leads to the desired amplitude. All other one- and two-fermion-line cases are related to the ones shown by parity or cyclic or reflection symmetries.

The one-fermion line, case (1), is trivial because $\mathcal{N}=1$ SYM forms a closed subsector of $\mathcal{N}=4$ SYM. In case (2a) we must choose all gluinos to have the same flavor; otherwise a scalar would be exchanged in the horizontal direction. Here, helicity conservation prevents the exchange of an unwanted gluon in this direction, keeping the two flavors distinct as desired. In case (2b), we must use two different gluino flavors, as shown; otherwise helicity conservation would allow gluon exchange in the wrong channel, corresponding to identical rather than distinct quarks.

More generally, in order to avoid scalar exchange, if two color-adjacent gluinos have the same helicity, then we should choose them to have the same flavor. In other words, we should forbid all configurations of the form $\left(\ldots, A^{+}, B^{+}, \ldots\right)$ and $\left(\ldots, A^{-}, B^{-}, \ldots\right)$ for $A \neq$ $B$, where $A^{ \pm}$stands for the gluino state $\tilde{g}_{A}^{ \pm}$. While this is necessary, it is not sufficient. For example, we also need to forbid configurations such as $\left(\ldots, A^{+}, C^{ \pm}, C^{\mp}, B^{+}, \ldots\right)$, because 


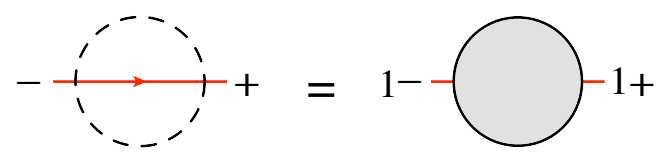

(2a)

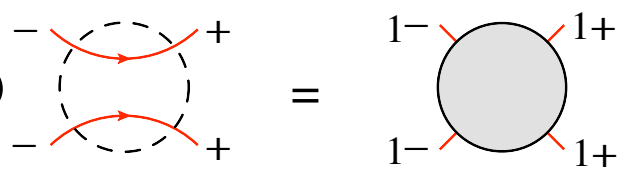

(2b)

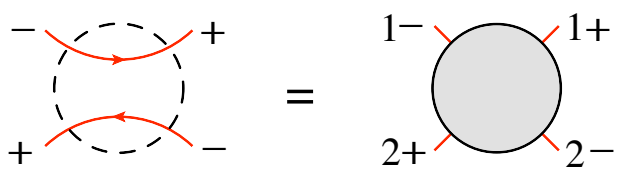

Figure 3. The possible fermion-line configurations for amplitudes with one fermion line, case (1), or two fermion lines, cases $(2 \mathrm{a})$ and $(2 \mathrm{~b})$.

(3a)

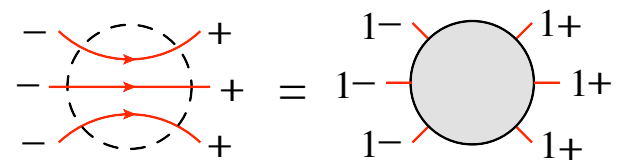

$(3 \mathrm{c})+$

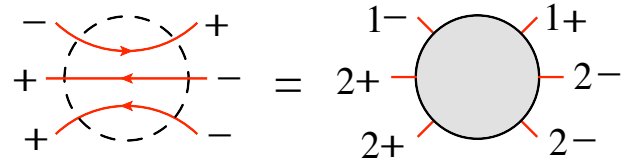

$(3 e)$

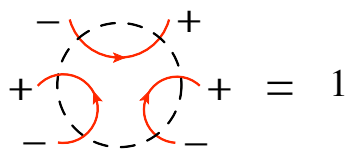

(3b)

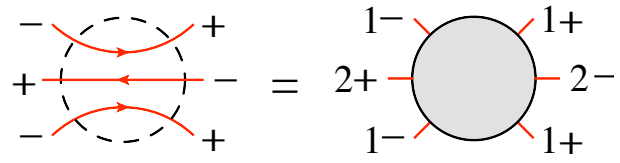

(3d)
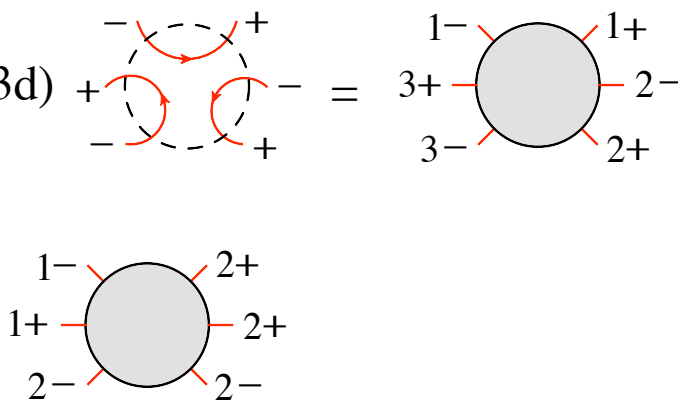

Figure 4. The possible fermion-line configurations for amplitudes with three fermion lines.

the pair $\left(C^{ \pm}, C^{\mp}\right)$ could be produced by a gluon splitting into this pair, which also connects to the $\left(A^{+}, B^{+}\right)$fermion line. As a secondary consideration, if two color-adjacent gluinos have opposite helicity, then we should choose them to have the same flavor or different flavor according to the desired quark flavor flow on the left-hand side. However, it will not always be possible to choose them to have a different flavor.

With these properties in mind, we can now examine figure 4, which shows solutions for the three-fermion-line cases. (Again, all other three-fermion-line cases are related to the ones shown by parity or cyclic or reflection symmetries.) Using the properties of the vanishing vertices in figure 2 , it is straightforward to show that only the desired QCD tree amplitudes on the left are produced by the gluino assignments on the right. The most subtle case is (3e). The two pairs of adjacent identical-helicity quarks force all the gluino flavors to be the same. However, this choice does not result in three distinct fermion lines in the pattern desired. There is a "wrong" fermion-line configuration which, fortunately, coincides with a permutation of case (3c). Hence we can subtract off this solution, as the second term on the right-hand side of (3e).

We have also examined the cases with four different fermion lines. There are 16 cases, shown in figure 5, and each has a solution. Four of the cases, (4g), (4k), (4l) and (4n), 
(4a)

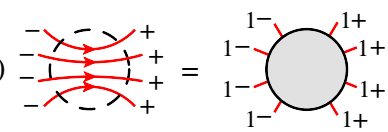

(4d)
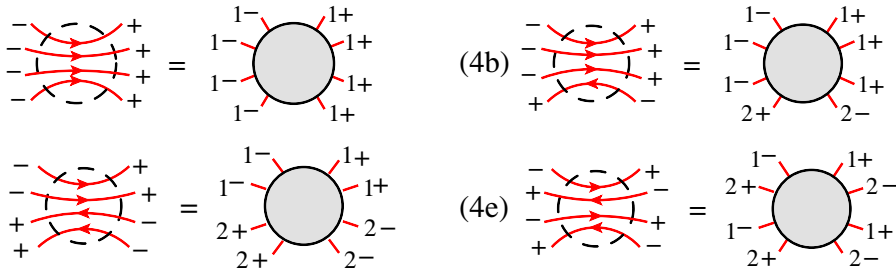

(4e)

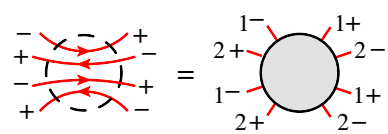

(4g)

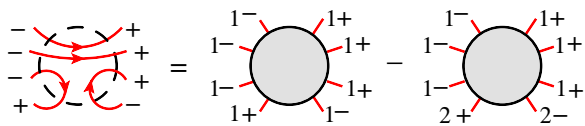

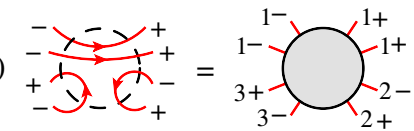

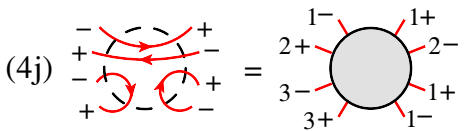

(4k)

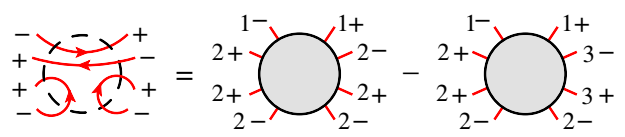

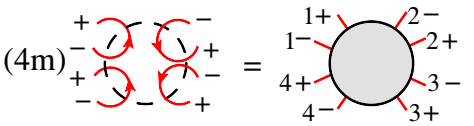

(41)

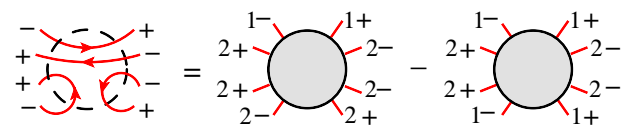

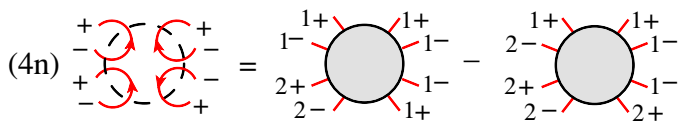

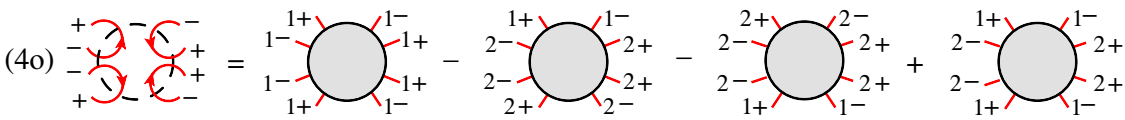

(4p)

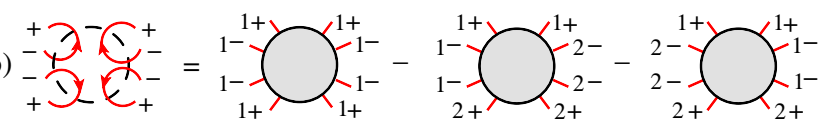

Figure 5. The possible fermion-line configurations for amplitudes with four fermion lines.

require two different terms, analogous to case $(3 \mathrm{e})$. Case $(4 \mathrm{p})$ requires three terms to remove all identical fermions; and case (4o) requires four separate terms. Case (4o) can be written as

$$
\begin{aligned}
A^{\mathrm{QCD}} & \left(\bar{q}_{1}^{-}, q_{1}^{+}, \bar{q}_{2}^{-}, q_{2}^{+}, q_{3}^{+}, \bar{q}_{3}^{-}, q_{4}^{+}, \bar{q}_{4}^{-}\right)=A^{\mathcal{N}=4 S Y M}\left(1^{-}, 1^{+}, 1^{-}, 1^{+}, 1^{+}, 1^{-}, 1^{+}, 1^{-}\right) \\
& -\left[A^{\mathcal{N}=4 S Y M}\left(2^{-}, 1^{+}, 1^{-}, 2^{+}, 2^{+}, 2^{-}, 2^{+}, 2^{-}\right)-A^{\mathcal{N}=4 S Y M}\left(2^{-}, 1^{+}, 1^{-}, 2^{+}, 2^{+}, 1^{-}, 1^{+}, 2^{-}\right)\right] \\
& -\left[A^{\mathcal{N}=4 S Y M}\left(2^{-}, 2^{+}, 2^{-}, 2^{+}, 2^{+}, 1^{-}, 1^{+}, 2^{-}\right)-A^{\mathcal{N}=4 S Y M}\left(2^{-}, 1^{+}, 1^{-}, 2^{+}, 2^{+}, 1^{-}, 1^{+}, 2^{-}\right)\right] \\
& -A^{\mathcal{N}=4 S Y M}\left(2^{-}, 1^{+}, 1^{-}, 2^{+}, 2^{+}, 1^{-}, 1^{+}, 2^{-}\right) \\
= & A^{\mathcal{N}=4 S Y M}\left(1^{-}, 1^{+}, 1^{-}, 1^{+}, 1^{+}, 1^{-}, 1^{+}, 1^{-}\right)-A^{\mathcal{N}=4 S Y M}\left(2^{-}, 1^{+}, 1^{-}, 2^{+}, 2^{+}, 2^{-}, 2^{+}, 2^{-}\right) \\
& -A^{\mathcal{N}=4 S Y M}\left(2^{-}, 2^{+}, 2^{-}, 2^{+}, 2^{+}, 1^{-}, 1^{+}, 2^{-}\right)+A^{\mathcal{N}=4 S Y M}\left(2^{-}, 1^{+}, 1^{-}, 2^{+}, 2^{+}, 1^{-}, 1^{+}, 2^{-}\right) .
\end{aligned}
$$

The first form of this equation indicates that three different wrong-fermion-line configurations have to be removed. However, all removals can be accomplished with the help of 
different permutations of two other cases, (4f) and (4l) respectively,

$$
\begin{aligned}
& A^{\mathrm{QCD}}\left(\bar{q}_{1}^{-}, q_{1}^{+}, \bar{q}_{2}^{-}, \bar{q}_{3}^{-}, q_{4}^{+}, \bar{q}_{4}^{-}, q_{3}^{+}, q_{2}^{+}\right)=A^{\mathcal{N}=4 S Y M}\left(1^{-}, 1^{+}, 2^{-}, 2^{-}, 1^{+}, 1^{-}, 2^{+}, 2^{+}\right), \\
& A^{\mathrm{QCD}}\left(\bar{q}_{1}^{-}, q_{1}^{+}, \bar{q}_{2}^{-}, \bar{q}_{3}^{-}, q_{3}^{+}, \bar{q}_{4}^{-}, q_{4}^{+}, q_{2}^{+}\right) \\
& =A^{\mathcal{N}=4 S Y M}\left(1^{-}, 1^{+}, 2^{-}, 2^{-}, 2^{+}, 2^{-}, 2^{+}, 2^{+}\right)-A^{\mathcal{N}=4 S Y M}\left(1^{-}, 1^{+}, 2^{-}, 2^{-}, 1^{+}, 1^{-}, 2^{+}, 2^{+}\right) \text {. }
\end{aligned}
$$

Case (4l) itself requires a wrong-fermion-line subtraction.

We have not yet ascertained whether any QCD tree amplitudes with more than four fermion lines are impossible to extract from $\mathcal{N}=4$ super Yang-Mills theory. Fortunately, for a fixed number of external partons, as one increases the number of fermion lines the number of Feynman diagrams decreases. Also, amplitudes with many external quarks typically contribute much less to a multi-jet cross section than do amplitudes with more gluons and fewer quarks.

Finally, we remark on the conversion of pure-QCD tree amplitudes, that is amplitudes for quarks and gluons, into amplitudes that contain in addition a single electroweak vector boson, namely a $W, Z$ or virtual photon. It is sufficient to compute the amplitude including the decay of the boson to a fermion-anti-fermion pair.

Consider first the case of a virtual photon which is emitted from a quark $q$ and decays to a charged lepton (Drell-Yan) pair $\ell^{+} \ell^{-}$. We can extract this amplitude from a colorordered amplitude with four consecutive fermions, as follows:

$$
A^{\gamma^{*}}\left(\ldots, q^{+}, \ell^{-}, \ell^{+}, \bar{q}^{-}, \ldots\right)=A^{\mathrm{QCD}}\left(\ldots, q_{1}^{+}, \bar{q}_{2}^{-}, q_{2}^{+}, \bar{q}_{1}^{-}, \ldots\right) .
$$

The color-ordering prevents gluons from being emitted from the quark line $q_{2}$, or from the virtual gluon connecting $q_{1}$ and $q_{2}$. Hence this virtual gluon is functionally identical to a virtual photon. The only other modification comes when dressing $A^{\gamma^{*}}$ with couplings. There is a relative factor of $2\left(-Q^{q}\right) e^{2} / g^{2}$ when doing so, where the " 2 " is related to the standard normalizations of the QED interaction with coupling $e$, versus the QCD interaction with coupling $g$, and $Q^{q}$ is the electric charge of the quark $q$. (The lepton has charge -1.)

It is possible to extract the amplitude (3.6) a second way, using one quark flavor instead of two,

$$
A^{\gamma^{*}}\left(\ldots, q^{+}, \ell^{-}, \ell^{+}, \bar{q}^{-}, \ldots\right)=-A^{\mathrm{QCD}}\left(\ldots, q_{1}^{+}, q_{1}^{+}, \bar{q}_{1}^{-}, \bar{q}_{1}^{-}, \ldots\right) .
$$

This alternative works because the color-ordered interaction for $g^{*} \rightarrow q^{+} \bar{q}^{-}$is antisymmetric under exchange of $q$ and $\bar{q}$, and because the exchange of a gluon between identical-flavor quarks in the wrong channel is prevented by helicity conservation.

If the virtual photon decays to other charged massless fermions, i.e. to a quark-antiquark pair $q^{\prime} \bar{q}^{\prime}$, the only difference is of course to use the appropriate quark charge, $-Q^{q} e^{2} \rightarrow Q^{q} Q^{q^{\prime}} e^{2}$. Because helicity amplitudes are used, it is also trivial to convert the virtual-photon amplitudes to ones for (parity-violating) electroweak boson production. For the case of combined exchange of virtual photon and $Z$ boson, with decay to a charged lepton pair, the electric charge factor has to be replaced by

$$
2 e^{2}\left(-Q^{q}+v_{L, R}^{\ell} v_{L, R}^{q} \mathcal{P}_{Z}\left(s_{\ell \bar{\ell}}\right)\right),
$$


where $v_{L, R}^{\ell}$ are the left- and right-handed couplings of the lepton to the $Z$ boson, $v_{L, R}^{q}$ are the corresponding quantities for the quark,

$$
\mathcal{P}_{Z}(s)=\frac{s}{s-M_{Z}^{2}+i \Gamma_{Z} M_{Z}}
$$

is the ratio of $Z$ to $\gamma^{*}$ propagators, and $M_{Z}$ and $\Gamma_{Z}$ are the mass and width of the $Z$.

Whether $v_{L}$ or $v_{R}$ is to be used in eq. (3.8) depends on the helicity assignment, i.e. on whether the $Z$ couples to a left- or right-handed outgoing fermion (as opposed to antifermion); see ref. [52] for further details. The case of a $W^{ \pm}$boson is even simpler, because there is no coupling to right-handed fermions (and no interference with another boson).

The relevant MHV and NMHV amplitudes for four external fermions and the rest gluons, and for six external fermions and the rest gluons, have been converted in the above manner into tree amplitudes for $V q \bar{q} g \ldots g$ and $V q \bar{q} Q \bar{Q} g \ldots g$, where $V$ stands for $W, Z$ or $\gamma^{*}$. These NMHV amplitudes have been incorporated into the BLACKHAT library [42] and used there in conjunction with a numerical implementation [53] of the BCFW (on-shell) recursion relations $[24,25]$ in order to obtain amplitudes at the NNMHV level and beyond. Including the MHV and NMHV formulae speeds up the numerical recursive algorithm by a factor of about four, in the present implementation. This approach was used to compute the real-radiative corrections entering the recent evaluation of $p p \rightarrow W+4$ jets at NLO [35], in particular the tree amplitudes for $W q \bar{q}^{\prime} g g g g g$ and $W q \bar{q}^{\prime} Q \bar{Q} g g g$. These amplitudes have nine external legs, after decaying the $W$ boson to a lepton pair, so there are MHV, NMHV and NNMHV configurations, but no further. All seven-point configurations are either MHV or NMHV, so at most two recursive steps were required to hit an explicit formula (for example, in a schematic notation $A_{9} \rightarrow A_{8} \times A_{3} \rightarrow A_{7} \times A_{3} \times A_{3}$ ).

We remark that the tree-level color-ordered amplitudes entering subleading-color loop amplitudes can have a more general color ordering from that required for purely tree-level applications. For example, in the pure QCD amplitudes with a single $q \bar{q}$ pair, only the colorordered amplitudes in which the two fermions are adjacent are needed in eq. (3.1). However, the subleading-color terms in the one-loop amplitudes for $q \bar{q} g \ldots g$ include many cases in which the two fermions are not color-adjacent, and the tree-level $q \bar{q} g \ldots g$ amplitudes that enter their unitarity cuts have the same property. These color-ordered amplitudes are all available in $\mathcal{N}=4$ super Yang-Mills theory, of course.

Similarly, for computing subleading-color one-loop terms for single-vector boson production processes like $V q \bar{q} g \ldots g$, one needs tree amplitudes such as $A^{\gamma^{*}}\left(\ldots, q^{+}, g, \ell^{-}, \ell^{+}\right.$, $\left.\bar{q}^{-}, \ldots\right)$, in which the gluon $g$ is color-ordered with respect to the quark pair, but not the lepton pair. These amplitudes are not equal to any particular color-ordered amplitude in $\mathcal{N}=4 \mathrm{SYM}$, but one can generate them by summing over appropriate color orderings. For example, we have

$$
\begin{aligned}
A^{\gamma^{*}}\left(\ldots, q^{+}, g, \ell^{-}, \ell^{+}, \bar{q}^{-}, \ldots\right)=A^{\mathrm{QCD}}\left(\ldots, q_{1}^{+}, g, \bar{q}_{2}^{-}, q_{2}^{+}, \bar{q}_{1}^{-}, \ldots\right) \\
\quad+A^{\mathrm{QCD}}\left(\ldots, q_{1}^{+}, \bar{q}_{2}^{-}, g, q_{2}^{+}, \bar{q}_{1}^{-}, \ldots\right)+A^{\mathrm{QCD}}\left(\ldots, q_{1}^{+}, \bar{q}_{2}^{-}, q_{2}^{+}, g, \bar{q}_{1}^{-}, \ldots\right) .
\end{aligned}
$$

The sum over the three permutations properly cancels out the unwanted poles as $g$ becomes collinear with either $\ell^{-}$or $\ell^{+}$. 


\section{All gluon tree amplitudes}

In this section we present the general expression for an $n$-gluon tree amplitude, which we derive in section 7 from the solution of ref. [29] for a general $\mathcal{N}=4$ SYM super-amplitude.

Amplitudes for $n$-gluon scattering are classified by the number of negative-helicity gluons occurring in them. Tree-amplitudes with fewer than two negative-helicity gluons vanish. In our conventions the gluon at position $n$ is always of negative helicity, which does not present a restriction due to cyclicity of the color-ordered amplitude. The ParkeTaylor formula [17] for a maximally-helicity-violating (MHV) gluon amplitude, with the two negative-helicity gluons sitting at positions $c_{0} \in[1, n-1]$ and $n$, then reads

$$
A_{n}\left(1^{+}, \ldots,\left(c_{0}-1\right)^{+}, c_{0}^{-},\left(c_{0}+1\right)^{+}, \ldots,(n-1)^{+}, n^{-}\right):=A_{n}^{\mathrm{MHV}}\left(c_{0}, n\right)=\frac{\delta^{(4)}(p)\left\langle c_{0} n\right\rangle^{4}}{\langle 12\rangle\langle 23\rangle \ldots\langle n 1\rangle}
$$

with the total conserved momentum $p=\sum_{i=1}^{n} p_{i}$.

Next-to-maximally-helicity-violating amplitudes of degree $p\left(\mathrm{~N}^{\mathrm{P}} \mathrm{MHV}\right)$ then consist of $(p+2)$ negative-helicity gluons embedded in $(n-p-2)$ positive-helicity states. We parametrize the positions of the negative-helicity gluons in the ordered set $\left(c_{0}, c_{1}, \ldots, c_{p}, n\right)$ with $c_{i} \in[1, n-1]$.

The general $\mathrm{N}^{\mathrm{p}} \mathrm{MHV}$ tree-amplitude then takes the form

$$
\begin{aligned}
& A_{n}^{\mathrm{Np} M H V}\left(c_{0}, c_{1}, \ldots, c_{p}, n\right)=\frac{\delta^{(4)}(p)}{\langle 12\rangle\langle 23\rangle \ldots\langle n 1\rangle} \times \\
& \quad \times \sum_{\text {all paths of length } p} 1 \cdot \tilde{R}_{n ; a_{1} b_{1}} \cdot \tilde{R}_{n ;\left\{I_{2}\right\} ; a_{2} b_{2}}^{\left.\left\{L_{2}\right\} ; \ldots U_{2}\right\}} \cdot \ldots \cdot \tilde{R}_{n ;\left\{I_{p}\right\} ; a_{p} b_{p}}^{\left.\left\{L_{p}\right\} ; U_{p}\right\}} \cdot\left(\operatorname{det} \Xi_{n}^{\text {path }}\left(c_{0}, \ldots, c_{p}\right)\right)^{4}
\end{aligned}
$$

Let us now explain in turn the ingredients of this result, i.e. the sum over paths, the $\tilde{R}$-functions, and the path-matrix $\Xi_{n}^{\text {path }}$.

The sum over all paths refers to the rooted tree depicted in figure 6 , introduced in ref. [29]. A path of length 0 consists of the initial node "1". A path of length $p$ leads to a sequence of $p+1$ nodes visited starting with node " 1 ". To clarify this all possible paths up to length $p=3$ are listed in figure 6 . In general there are $(2 p) ! /(p !(p+1) !)$ different paths of length $p$, which is equal to the number of nodes appearing at level $p$ in the rooted tree, since each final node unambiguously determines a path through the rooted tree.

The $\tilde{R}$-functions are generalizations of eq. (2.8) and may be written rather compactly with the help of eq. (2.6) as

$$
\tilde{R}_{n ;\{I\} ; a b}:=\frac{1}{x_{a b}^{2}} \frac{\langle a(a-1)\rangle}{\langle n\{I\} b a \mid a\rangle\langle n\{I\} b a \mid a-1\rangle} \frac{\langle b(b-1)\rangle}{\langle n\{I\} a b \mid b\rangle\langle n\{I\} a b \mid b-1\rangle} ;
$$

they derive from the dual superconformal $R$-invariants introduced in ref. [29]. In the above and in eq. (4.2), $\{I\}$ is a multi-index deriving from the node in the associated path with the last pair of indices stripped off, e.g. $\left\{I_{3}\right\}=\left\{b_{1}, a_{1}, b_{2}, a_{2}\right\}$ for the last node of the first path of length $p=3$. 


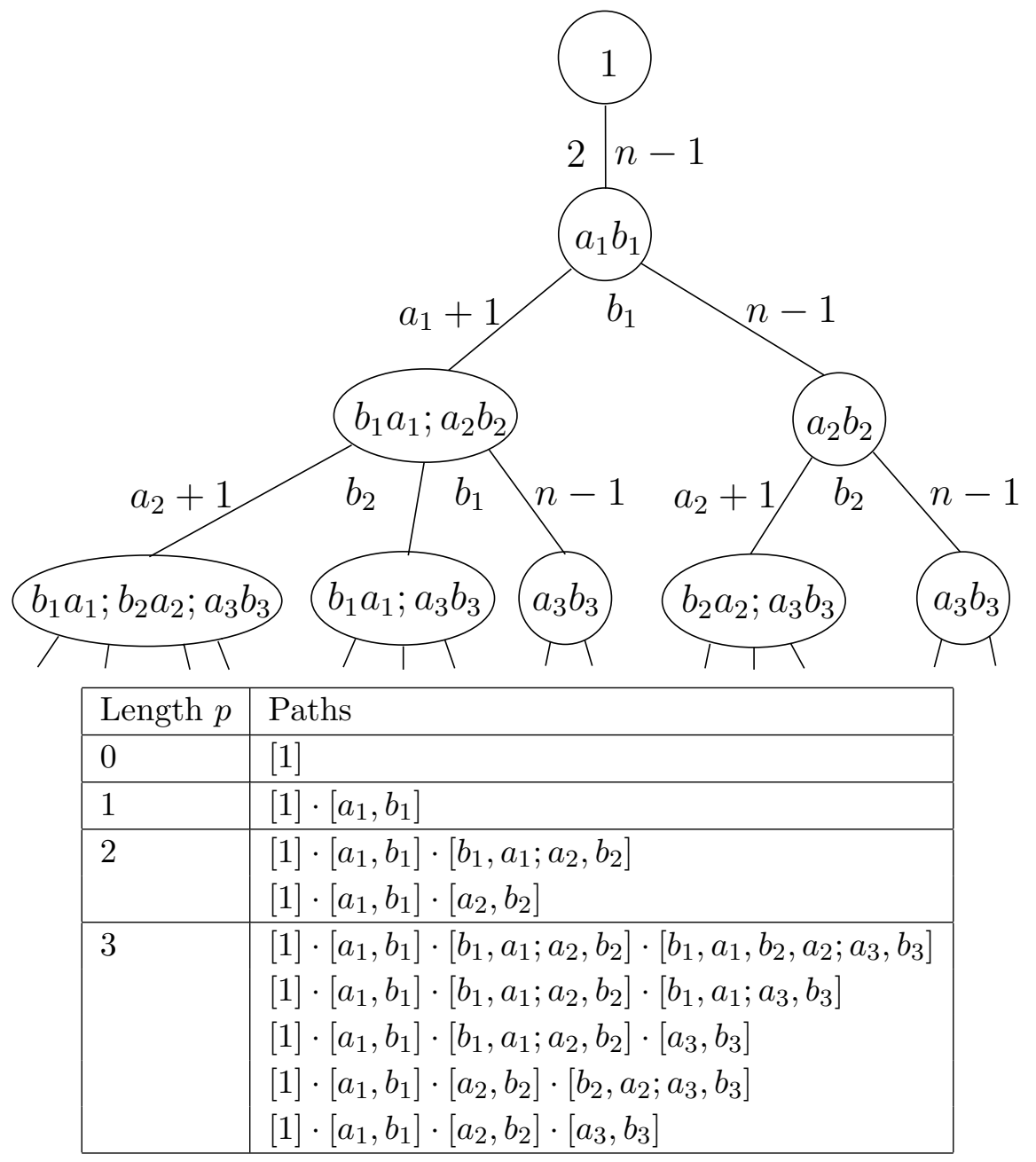

Figure 6. The rooted tree encoding the sum over paths occurring in eq. (4.2). The table shows all paths up to length 3 .

In eq. (4.2) we used a further piece of notation, namely $\tilde{R}$-functions with upper indices, which start to appear at the NNMHV level, and which we now define. Generally the $\tilde{R}$ functions appear in the amplitude with an ordered summation over the last pair of indices,

$$
\sum_{L \leq a<b \leq U} \tilde{R}_{n ;\{I\} ; a b}
$$

$\tilde{R}$-functions with upper indices indicate a special behavior for the boundary terms in this sum when $a=L$ or $b=U$. Specifically if one has

$$
\sum_{L \leq a<b \leq U} \tilde{R}_{n ;\{I\} ; a b}^{l_{1}, \ldots, l_{p} ; u_{1}, \ldots, u_{q}}
$$

and the boundary of a summation is reached, then the occurrence of the spinor $|a-1\rangle$ or $|b\rangle$ in the $\tilde{R}$-function without upper indices (4.3) is replaced by a novel spinor depending 
on the upper indices as follows

$$
\begin{array}{rlr}
\langle L-1| & \longrightarrow\left\langle\xi_{L}\right|:=\langle n| x_{n l_{1}} x_{l_{1} l_{2}} \ldots x_{l_{p-1} l_{p}} & \text { for } a=L, \\
\langle U| & \longrightarrow\left\langle\xi_{U}\right|:=\langle n| x_{n u_{1}} x_{u_{1} u_{2}} \ldots x_{u_{q-1} u_{q}} & \text { for } b=U .
\end{array}
$$

Effectively this amounts to the following formula for the upper-indexed $\tilde{R}$-functions of eq. (4.5),

$$
\tilde{R}_{n ;\{I\} ; a b}^{l_{1}, \ldots, l_{p} ; u_{1}, \ldots, u_{q}}= \begin{cases}\tilde{R}_{n ;\{I\} ; a b} \cdot \frac{\left\langle a \xi_{L}\right\rangle}{\left\langle n\{I\} b a \mid \xi_{L}\right\rangle} \frac{\langle n\{I\} b a \mid a-1\rangle}{\langle a(a-1)\rangle} & \text { for } a=L, \\ \tilde{R}_{n ;\{I\} ; a b} \cdot \frac{\left\langle\xi_{U}(b-1)\right\rangle}{\left.\langle n\{1\} a b) \xi_{U}\right\rangle} \frac{\langle n\{I\} a b \mid b\rangle}{\langle(b(b-1)\rangle} & \text { for } b=U, \\ \tilde{R}_{n ;\{I\} ; a b} \cdot \frac{\left\langle a \xi_{L}\right\rangle}{\left\langle n\{I\} b a \mid \xi_{L}\right\rangle} \frac{\langle n\{I\} b a \mid a-1\rangle}{\langle a(a-1)\rangle} \frac{\left\langle\xi_{U}(b-1)\right\rangle}{\left\langle n\{I\} a b \mid \xi_{U}\right\rangle} \frac{\langle n\{I\} a b \mid b\rangle}{\langle b(b-1)\rangle} & \text { for } a=L \text { and } b=U, \\ \tilde{R}_{n ;\{I\} ; a b} & \text { else. }\end{cases}
$$

In particular there is a term in the double sum where both $a=L$ and $b=U$ are reached and both replacements are to be made. The rules for constructing the sets of upper indices $l_{1}, \ldots, l_{p} ; u_{1}, \ldots, u_{q}$ in eq. (4.5) from the rooted tree are given in ref. [29].

To write down the path-matrix $\Xi_{n}^{\text {path }}$ we furthermore need to define the quantities

$$
\begin{aligned}
\left(\Xi_{n}\right)_{0}^{c_{i}} & :=\left\langle n c_{i}\right\rangle \\
\left(\Xi_{n}\right)_{a b}^{c_{i}} & :=\left\langle n b a \mid c_{i}\right\rangle \chi_{[a, b-1]}\left(c_{i}\right)-x_{a b}^{2}\left\langle n c_{i}\right\rangle \chi_{[b, n-1]}\left(c_{i}\right) \\
\left(\Xi_{n}\right)_{\left\{b_{1}, a_{1}, \ldots, b_{r}, a_{r}\right\} ; a b}^{c_{c_{i}}} & :=\left\langle n b_{1} a_{1} \ldots b_{r} a_{r} a b \mid c_{i}\right\rangle \chi_{[a, b-1]}\left(c_{i}\right)-x_{a b}^{2}\left\langle n b_{1} a_{1} \ldots b_{r} a_{r} \mid c_{i}\right\rangle \chi_{\left[a_{r}, a-1\right]}\left(c_{i}\right),
\end{aligned}
$$

with the support functions

$$
\chi_{[a, b]}(i)= \begin{cases}1 & \text { if } i \in[a, b] \\ 0 & \text { else. }\end{cases}
$$

Now to every node $[\{I\} ; a b]$ along a given path and to every negative-helicity leg $c_{i}$ we associate the entry of the path-matrix $\left(\Xi_{n}\right)_{\{I\} ; a b}^{c_{i}}$. The entries $\left(\Xi_{n}\right)^{A}{ }_{B}$ form a $(p+1) \times(p+1)$ matrix. Explicitly one has

$$
\Xi_{n}^{\text {path }}\left(c_{0}, \ldots, c_{p}\right):=\left(\begin{array}{lll}
\left\langle n c_{0}\right\rangle & \left\langle n c_{1}\right\rangle & \ldots\left\langle n c_{p}\right\rangle \\
\left(\Xi_{n}\right)_{a_{1} b_{1}}^{c_{0}} & \left(\Xi_{n}\right)_{a_{1} b_{1}}^{c_{1}} & \ldots\left(\Xi_{n}\right)_{a_{1} b_{1}}^{c_{p}} \\
\left(\Xi_{n}\right)_{\left\{I_{2}\right\} ; a_{2} b_{2}}^{c_{0}} & \left(\Xi_{n}\right)_{\left\{I_{2}\right\} ; a_{2} b_{2}}^{c_{1}} \ldots\left(\Xi_{n}\right)_{\left\{I_{2}\right\} ; a_{2} b_{2}}^{c_{p}} \\
\vdots & \vdots & \vdots \\
\left(\Xi_{n}\right)_{\left\{I_{p}\right\} ; a_{p} b_{p}}^{c_{0}} & \left(\Xi_{n}\right)_{\left\{I_{p}\right\} ; a_{p} b_{p}}^{c_{1}} \ldots\left(\Xi_{n}\right)_{\left\{I_{p}\right\} ; a_{p} b_{p}}^{c_{p}}
\end{array}\right) .
$$

Although $\tilde{R}$ and $\Xi_{n}^{\text {path }}$ look rather involved at first sight, they are determined entirely through the external spinors $\lambda_{i}$ and $\tilde{\lambda}_{i}$. 
To clarify the construction principle let us write down the first three amplitudes in the $\mathrm{N}^{\mathrm{P}} \mathrm{MHV}$ series explicitly:

$$
\begin{aligned}
& A_{n}^{\mathrm{MHV}}\left(c_{0}, n\right)=\frac{\delta^{(4)}(p)}{\langle 12\rangle\langle 23\rangle \ldots\langle n 1\rangle} \cdot\left\langle n c_{0}\right\rangle^{4}, \\
& A_{n}^{\mathrm{NMHV}}\left(c_{0}, c_{1}, n\right)=\frac{\delta^{(4)}(p)}{\langle 12\rangle\langle 23\rangle \ldots\langle n 1\rangle} \sum_{2 \leq a_{1}<b_{1} \leq n-1} \tilde{R}_{n ; a_{1} b_{1}} \cdot\left|\begin{array}{ll}
\left\langle n c_{0}\right\rangle & \left\langle n c_{1}\right\rangle \\
\left(\Xi_{n}\right)_{a_{1} b_{1}}^{c_{0}} & \left(\Xi_{n}\right)_{a_{1} b_{1}}^{c_{1}}
\end{array}\right|^{4}, \\
& A_{n}^{\mathrm{N}^{2} \mathrm{MHV}}\left(c_{0}, c_{1}, c_{2}, n\right)=\frac{\delta^{(4)}(p)}{\langle 12\rangle\langle 23\rangle \ldots\langle n 1\rangle} \sum_{2 \leq a_{1}<b_{1}<n} \tilde{R}_{n ; a_{1} b_{1}}
\end{aligned}
$$

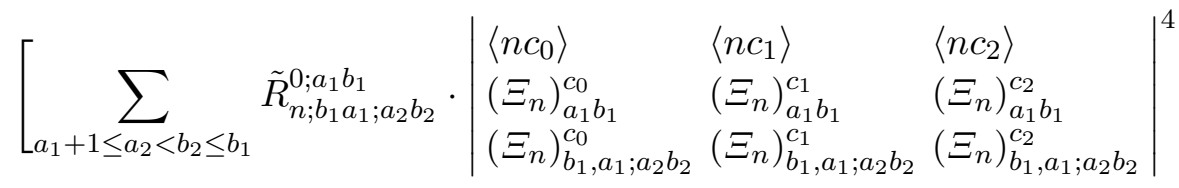

$$
\begin{aligned}
& \left.+\sum_{b_{1} \leq a_{2}, b_{2}<n} \tilde{R}_{n ; a_{2} b_{2}}^{a_{1} b_{1} ; 0} \cdot\left|\begin{array}{lll}
\left\langle n c_{0}\right\rangle & \left\langle n c_{1}\right\rangle & \left\langle n c_{2}\right\rangle \\
\left(\Xi_{n}\right)_{a_{1} b_{1}}^{c_{0}} & \left(\Xi_{n}\right)_{a_{1} b_{1}}^{c_{1}} & \left(\Xi_{n}\right)_{a_{1} b_{1}}^{c_{2}} \\
\left(\Xi_{n}\right)_{a_{2} b_{2}}^{c_{0}} & \left(\Xi_{n}\right)_{a_{2} b_{2}}^{c_{1}} & \left(\Xi_{n}\right)_{a_{2} b_{2}}^{c_{2}}
\end{array}\right|^{4}\right]
\end{aligned}
$$

In appendix A we spell out the NMHV and $\mathrm{N}^{2} \mathrm{MHV}$ amplitudes explicitly. We provide a Mathematica package GGT with the arXiv .org submission of this article, which expands the master formula (4.2) explicitly for any choice of $p$, positions $c_{i}$ and momenta $\lambda_{i} \tilde{\lambda}_{i}$. See appendix $\mathrm{C}$ for documentation. The formula (4.2) is implemented by the function GGTgluon.

It should be mentioned that in practice the number of terms arising from the determinants of the path-matrix $\Xi_{n}^{\text {path }}$ is often quite small, see e.g. eq. (2.7). Moreover, for small $n$ the number of non-zero terms in the nested sums can be relatively small.

\section{All single-flavor quark-anti-quark-gluon trees}

Turning to the gauge-theory amplitudes involving massless single-flavor quark-anti-quark pairs we can write down a similarly general formula based on paths along the rooted tree of figure 6. In an abuse of notation, we refer here to a helicity $+\frac{1}{2}$ fermion as a quark, and a helicity $-\frac{1}{2}$ fermion as an anti-quark. Looking at a color-ordered $n$-parton amplitude involving gluons and $k$ quark-anti-quark pairs, $g^{n-2 k}(q \bar{q})^{k}$, it is again classified as a NPMHV amplitude by the number $(2+p-k)$ of negative-helicity gluons. In such a color-ordered amplitude we furthermore consider an arbitrary ordering of the fermions. We then have $2+p+k$ 'special' legs (negative-helicity gluon, quark or anti-quark) in such an amplitude, whose position amongst the $n$ legs we parametrize by the set

$$
\left(c_{0}, \ldots, c_{\alpha_{1}}, \ldots, c_{\bar{\beta}_{1}} \ldots, c_{\alpha_{k}}, \ldots, c_{\bar{\beta}_{k}}, \ldots, c_{p+k}, n\right) .
$$

Here $c_{i}$ denotes the position of a negative-helicity gluon, $c_{\alpha_{j}}$ a quark and $c_{\bar{\beta}_{j}}$ an anti-quark location. Note that while the quark and anti-quark locations $c_{\alpha_{i}}$ and $c_{\bar{\beta}_{i}}$ are considered as ordered sets, i.e. $c_{\alpha_{i}}<c_{\alpha_{j}}$ and $c_{\bar{\beta}_{i}}<c_{\bar{\beta}_{j}}$ for $i<j$, there is no such ordering in the total set 
$\left\{c_{\alpha_{i}}, c_{\bar{\beta}_{i}}\right\}$ reflecting an arbitrary sequence of quarks and anti-quarks in the color-ordered amplitude. Again in our convention one negative-helicity gluon is always located on leg $n{ }^{1}$

The general $\mathrm{N}^{\mathrm{P}} \mathrm{MHV}$ tree-amplitude for such a configuration then reads

$$
\begin{aligned}
& A_{(q \bar{q})^{k}, n}^{\mathrm{NPMHV}}(\left.c_{0}, \ldots, c_{\alpha_{1}}, \ldots, c_{\bar{\beta}_{1}}, \ldots, c_{\alpha_{k}}, \ldots, c_{\bar{\beta}_{k}}, \ldots, c_{p+k}, n\right)=\frac{\delta^{(4)}(p)}{\langle 12\rangle\langle 23\rangle \ldots\langle n 1\rangle} \times \\
& \times \operatorname{sign}(\tau) \sum_{\begin{array}{c}
\text { all paths } \\
\text { of length } p
\end{array}}\left(\prod_{i=1}^{p} \tilde{R}_{n ;\left\{I_{i}\right\} ; a_{i} b_{i}}^{\left\{L_{i}\right\} ;\left\{U_{i}\right\}}\right) \operatorname{det}\left(\left.\Xi_{n}^{\text {path }}\right|_{q}\right)^{3} \operatorname{det}\left(\left.\Xi_{n}^{\text {path }}(\bar{q} \leftrightarrow q)\right|_{\bar{q}}\right) .
\end{aligned}
$$

Here $\operatorname{sign}(\tau)$ is the sign produced by permuting the quark and anti-quark legs into the alternating order $\left\{c_{\alpha_{1}}, c_{\bar{\beta}_{1}}, c_{\alpha_{2}}, c_{\bar{\beta}_{2}}, \ldots, c_{\alpha_{k}}, c_{\bar{\beta}_{k}}\right\}$.

Remarkably, the only difference from the pure gluon amplitudes is a change in the determinant factors of the path-matrix $\Xi_{n}^{\text {path }}$. With $2+p+k$ 'special' legs the path-matrix associated to eq. (5.1) is now a $(p+1) \times(p+1+k)$ matrix of the form

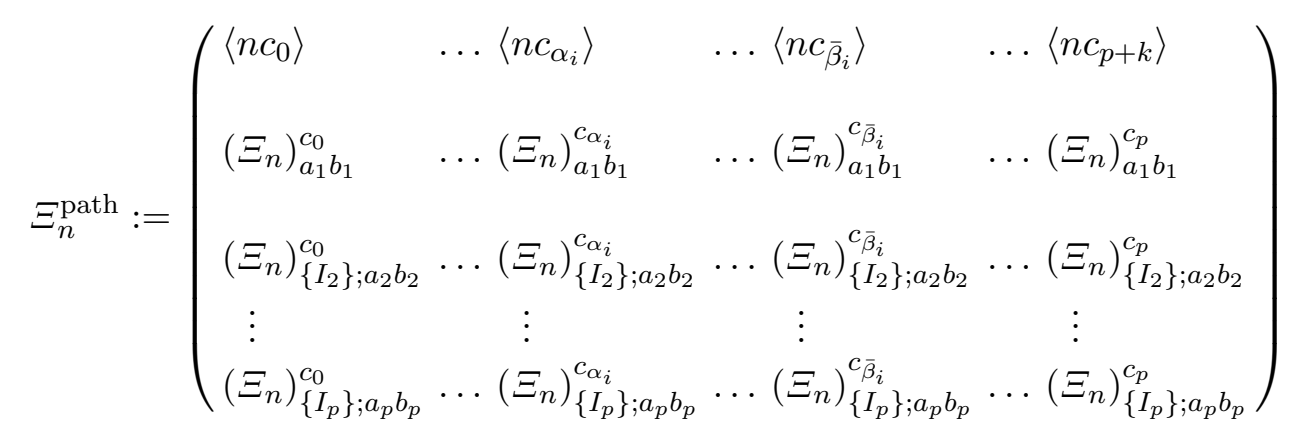

The notation $\left.\Xi_{n}^{\text {path }}\right|_{q}$ in eq. (5.2) now refers to the elimination of all the quark columns (the $c_{\alpha_{i}}$ 's) in the path-matrix and $\left.\Xi_{n}^{\text {path }}(\bar{q} \leftrightarrow q)\right|_{\bar{q}}$ denotes the matrix with all the anti-quark columns removed (the $c_{\bar{\beta}_{i}}$ 's) after quark and anti-quark columns have been interchanged, i.e. $c_{\bar{\beta}_{i}} \leftrightarrow c_{\alpha_{i}}$. The removal of $k$ columns is of course necessary in order to turn the $(p+1+k) \times(p+1)$ matrix $\Xi_{n}^{\text {path }}$ into square form, so that the determinant is defined.

Let us again spell out some of the lower $p$ amplitudes explicitly to clarify the formula (5.2):

$$
\begin{aligned}
A_{q \bar{q}, n}^{\mathrm{MHV}}\left(c_{\alpha_{1}}, c_{\bar{\beta}_{1}}, n\right) & =\frac{\delta^{(4)}(p)}{\langle 12\rangle\langle 23\rangle \ldots\langle n 1\rangle} \cdot\left\langle n c_{\bar{\beta}_{1}}\right\rangle^{3} \cdot\left\langle n c_{\alpha_{1}}\right\rangle \\
A_{q \bar{q}, n}^{\mathrm{NMHV}}\left(c_{0}, c_{\alpha_{1}}, c_{\bar{\beta}_{1}}, n\right) & =\frac{\delta^{(4)}(p)}{\langle 12\rangle\langle 23\rangle \ldots\langle n 1\rangle} \sum_{2 \leq a_{1}<b_{1} \leq n-1} \tilde{R}_{n ; a_{1} b_{1}} \cdot \\
& \left|\begin{array}{ll}
\left\langle n c_{0}\right\rangle & \left\langle n c_{\bar{\beta}_{1}}\right\rangle \\
\left(\Xi_{n}\right)_{a_{1} b_{1}}^{c_{0}} & \left(\Xi_{n}\right)_{a_{1} b_{1}}
\end{array}\right|^{3} \cdot\left|\begin{array}{ll}
\left\langle n c_{0}\right\rangle & \left\langle n c_{\alpha_{1}}\right\rangle \\
\left(\Xi_{n}\right)_{a_{1} b_{1}}^{c_{0}} & \left(\Xi_{n}\right)_{a_{1} b_{1}}^{c_{\alpha_{1}}}
\end{array}\right|
\end{aligned}
$$

\footnotetext{
${ }^{1}$ We comment in section 7 on how to circumvent this problem for the case without a single negativehelicity gluon.
} 


$$
\begin{aligned}
& A_{(q \bar{q})^{2}, n}^{\mathrm{NMHV}}\left(c_{\alpha_{1}}, c_{\bar{\beta}_{1}}, c_{\alpha_{2}}, c_{\bar{\beta}_{2}}, n\right)=\frac{\delta^{(4)}(p)}{\langle 12\rangle\langle 23\rangle \ldots\langle n 1\rangle} \sum_{2 \leq a_{1}<b_{1} \leq n-1} \tilde{R}_{n ; a_{1} b_{1}} . \\
& \left|\begin{array}{ll}
\left\langle n c_{\bar{\beta}_{1}}\right\rangle & \left\langle n c_{\bar{\beta}_{2}}\right\rangle \\
\left(\Xi_{n}\right)_{a_{1} \bar{\beta}_{1}} & \left(\Xi_{n}\right)_{c_{\bar{\beta}_{1} b_{1}}}
\end{array}\right|^{3} \cdot\left|\begin{array}{ll}
\left\langle n c_{\alpha_{1}}\right\rangle & \left\langle n c_{\alpha_{2}}\right\rangle \\
\left(\Xi_{n}\right)_{a_{1} b_{1}}^{c_{\alpha_{1}}} & \left(\Xi_{n}\right)_{a_{1} b_{1}}^{c_{\alpha_{2}}}
\end{array}\right| \\
& A_{q \bar{q}, n}^{\mathrm{N}^{2} \mathrm{MHV}}\left(c_{\alpha_{1}}, c_{1}, c_{\bar{\beta}_{1}}, c_{2}, n\right)=\frac{\delta^{(4)}(p)}{\langle 12\rangle\langle 23\rangle \ldots\langle n 1\rangle} \sum_{2 \leq a_{1}<b_{1}<n} \tilde{R}_{n ; a_{1} b_{1}} .
\end{aligned}
$$

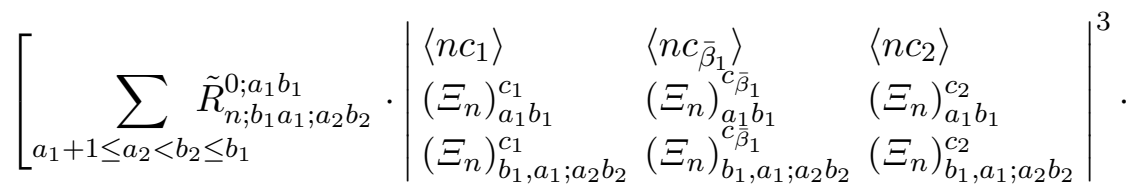

$$
\begin{aligned}
& \left|\begin{array}{lll}
\left\langle n c_{1}\right\rangle & \left\langle n c_{\alpha_{1}}\right\rangle & \left\langle n c_{2}\right\rangle \\
\left(\Xi_{n}\right)_{a_{1} b_{1}}^{c_{1}} & \left(\Xi_{n}\right)_{\alpha_{\alpha_{1}}} & \left(\Xi_{n}\right)_{a_{1} b_{1}}^{c_{2}} \\
\left(\Xi_{n}\right)_{b_{1}, a_{1} ; a_{2} b_{2}} & \left(\Xi_{n}\right)_{b_{\alpha_{1}}, a_{1} ; a_{2} b_{2}} & \left(\Xi_{n}\right)_{b_{1}, a_{1} ; a_{2} b_{2}}^{2}
\end{array}\right| \\
& \left.+\sum_{b_{1} \leq a_{2}, b_{2}<n} \tilde{R}_{n ; a_{2} b_{2}}^{a_{1} b_{1} ; 0} \cdot\left|\begin{array}{lll}
\left\langle n c_{1}\right\rangle & \left\langle n c_{\bar{\beta}_{1}}\right\rangle & \left\langle n c_{2}\right\rangle \\
\left(\Xi_{n}\right)_{a_{1} b_{1}}^{c_{1}} & \left(\Xi_{n}\right)_{c_{\bar{\beta}_{1}}} & \left(\Xi_{n}\right)_{a_{1} b_{1}}^{c_{2}} \\
\left(\Xi_{n}\right)_{a_{2} b_{2}}^{c_{1}} & \left(\Xi_{n}\right)_{a_{2} b_{1}} & \left(\Xi_{n}\right)_{a_{2} b_{2}}^{c_{2}}
\end{array}\right|^{3}\left|\begin{array}{lll}
\left\langle n c_{1}\right\rangle & \left\langle n c_{\alpha_{1}}\right\rangle & \left\langle n c_{2}\right\rangle \\
\left(\Xi_{n}\right)_{a_{1} b_{1}}^{c_{1}} & \left(\Xi_{n}\right)_{a_{\alpha_{1}}} & \left(\Xi_{n}\right)_{a_{2}}^{c_{2} b_{1}} \\
\left(\Xi_{n}\right)_{a_{2} b_{2}}^{c_{1}} & \left(\Xi_{n}\right)_{a_{2} b_{2}} & \left(\Xi_{n}\right)_{a_{2} b_{2}}^{c_{2} b_{2}}
\end{array}\right|\right] \\
& A_{(q \bar{q})^{2}, n}^{\mathrm{N}^{2} \mathrm{MHV}}\left(c_{\alpha_{1}}, c_{1}, c_{\bar{\beta}_{1}}, c_{\alpha_{2}}, c_{\bar{\beta}_{2}}, n\right)=\frac{\delta^{(4)}(p)}{\langle 12\rangle\langle 23\rangle \ldots\langle n 1\rangle} \sum_{2 \leq a_{1}<b_{1}<n} \tilde{R}_{n ; a_{1} b_{1}} .
\end{aligned}
$$

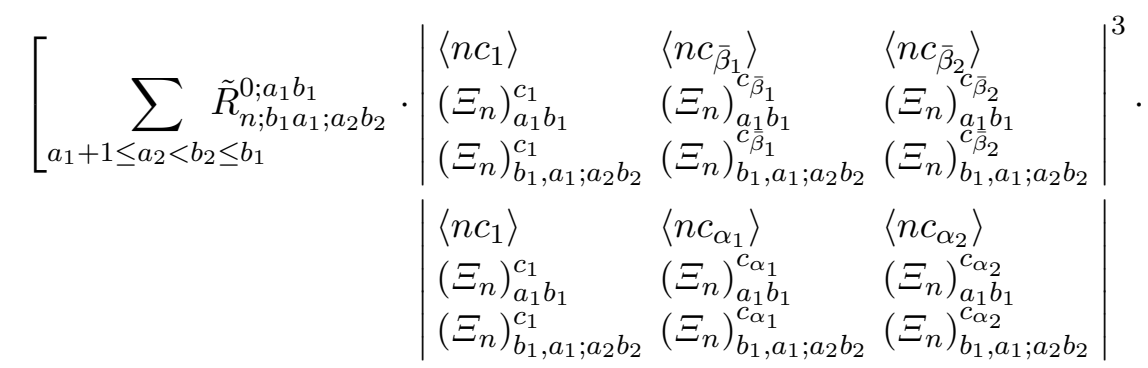

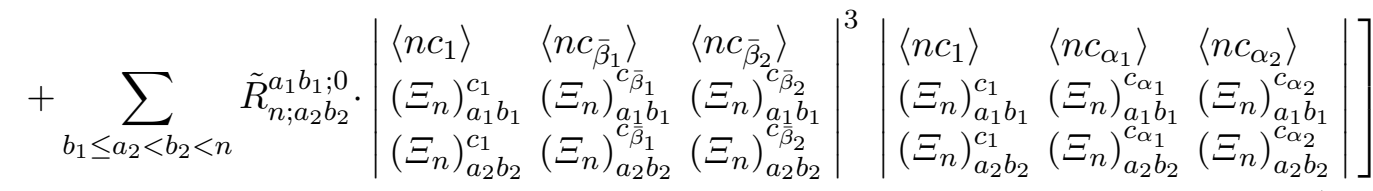

This completes our examples. Some explicit formulae with the determinants expanded out may be found in appendix B. The formula (5.2) is implemented in GGT by the function GGTfermions. See appendix C for the documentation.

Note that the master formula (4.2) reduces as it should to the pure-gluon scattering result (4.2) for a zero number of quark-anti-quark pairs, $k \rightarrow 0$. In that case no column removals are to be performed and the determinants combine to the power four. 


\section{All gluon-gluino tree amplitudes in $\mathcal{N}=4$ super Yang-Mills}

The color-ordered tree amplitudes with fermions presented above were special in the sense that they apply both to massless QCD as well as $\mathcal{N}=4$ super Yang-Mills, due to the single-flavor choice which suppresses intermediate scalar exchange as argued in section 3 . We now state the master formula for general gluino and gluon tree amplitudes in the $\mathcal{N}=4$ super Yang-Mills theory from which the above expressions arise. Through specific choices of external flavor configurations, however, this master formula may be used to produce color-ordered gluon and quark trees in massless QCD, as was discussed in section 3.

Similar to the notation in section 5, for a general $g^{n-2 k}(q \bar{q})^{k}$ amplitude with arbitrary flavor assignments to the 'quarks' 2 we have $2+p+k$ 'special' legs (negative-helicity gluon, quark or anti-quark), whose position amongst the $n$ legs we parametrize by the set

$$
\left(c_{0}, \ldots, c_{\alpha_{1}}^{A_{1}}, \ldots, c_{\bar{\beta}_{1}}^{B_{1}} \ldots, c_{\alpha_{k}}^{A_{k}}, \ldots, c_{\bar{\beta}_{k}}^{B_{k}}, \ldots, c_{p+k}, n\right) .
$$

Again the configuration of quarks and anti-quarks inside the gluon background may be arbitrary while the sets $\left\{\alpha_{i}\right\}$ and $\left\{\bar{\beta}_{i}\right\}$ are ordered. The general $g^{n-2 k}(q \bar{q})^{k}$ amplitude with $(2+p-k)$ negative-helicity gluons is then expressed in terms of the $\tilde{R}$-functions and the path-matrix $\Xi_{(q \bar{q})^{k}, n}^{\text {path }}$ defined above. It reads

$$
\begin{aligned}
& A_{(q \bar{q})^{k}, n}^{\mathrm{NPHV}}\left(c_{0}, \ldots, c_{\alpha_{i}}^{A_{i}}, \ldots, c_{\bar{\beta}_{j}}^{B_{j}} \ldots, c_{p+k}, n\right)=\frac{\delta^{(4)}(p) \operatorname{sign}(\tau)}{\langle 12\rangle\langle 23\rangle \ldots\langle n 1\rangle} \times \\
& \times \sum_{\substack{\text { all paths } \\
\text { of length } p}}\left(\prod_{i=1}^{p} \tilde{R}_{n ;\left\{I_{i}\right\} ; a_{i} b_{i}}^{\left.\left\{L_{i}\right\} ; U_{i}\right\}}\right)\left(\left.\operatorname{det} \Xi_{n}^{\text {path }}\right|_{q}\right)^{4-k} \times \\
& \times \sum_{\sigma \in S_{k}} \operatorname{sign}(\sigma) \prod_{i=1}^{k} \delta^{A_{i} B_{\sigma(i)}} \operatorname{det}\left(\left.\Xi_{n}^{\text {path }}\right|_{q}\left(\bar{\beta}_{\sigma(i)} \leftrightarrow \alpha_{i}\right)\right) .
\end{aligned}
$$

Here the notation $\left.\Xi_{n}^{\text {path }}\right|_{q}$ refers to the path-matrix (5.3) with all 'quark' columns $\left\{c_{\alpha_{i}}\right\}$ removed, whereas $\left.\Xi_{n}^{\text {path }}\right|_{q}\left(\bar{\beta}_{i} \rightarrow \alpha_{i}\right)$ denotes the same path-matrix where the 'anti-quark' column $c_{\bar{\beta}_{j}}$ is replaced by one of the previously-removed 'quark' columns $c_{\alpha_{i}}$. Also $\operatorname{sign}(\tau)$ is the sign of the permutation for bringing the initial color-ordering of the fermionic legs into the canonical order $\left\{c_{\alpha_{1}}, c_{\bar{\beta}_{1}}, c_{\alpha_{2}}, c_{\bar{\beta}_{2}}, \ldots, c_{\alpha_{k}}, c_{\bar{\beta}_{k}}\right\}$ and $\operatorname{sign}(\sigma)$ is the sign of the permutation of $\sigma$. This formula is implemented in GGT by the function GGTfermion.

\section{Proof of the master formula}

In this section we prove the master formula (6.2) for a general $\mathcal{N}=4$ super Yang-Mills tree amplitude with external gluons and gluinos of arbitrary flavor, as well as the more compact single-flavor expression (5.2) and the pure gluon expression (4.2) as sub-cases.

Amplitudes in $\mathcal{N}=4$ super Yang-Mills are very efficiently expressed in terms of a superwave function $\Phi$ which collects all on-shell states of the PCT self-conjugate massless

\footnotetext{
${ }^{2}$ We refer to the gluinos in this and the following sections as 'quarks' in order to not introduce new terminology beyond that introduced already in sections 4 and 5 .
} 
$\mathcal{N}=4$ multiplet, with the help of the Grassmann variables $\eta^{A}$ with $A=1,2,3,4$ of the su(4) R-symmetry,

$$
\begin{aligned}
\Phi(\lambda, \tilde{\lambda}, \eta)= & g^{+}(\lambda, \tilde{\lambda})+\eta^{A} \tilde{g}_{A}(\lambda, \tilde{\lambda})+\frac{1}{2} \eta^{A} \eta^{B} \phi_{A B}(\lambda, \tilde{\lambda})+\frac{1}{3 !} \eta^{A} \eta^{B} \eta^{C} \epsilon_{A B C D} \overline{\tilde{g}}^{D}(\lambda, \tilde{\lambda}) \\
& +\frac{1}{4 !} \eta^{A} \eta^{B} \eta^{C} \eta^{D} \epsilon_{A B C D} g^{-}(\lambda, \tilde{\lambda})
\end{aligned}
$$

Here $g^{ \pm}$are the \pm 1 helicity gluons, $\tilde{g}_{A}$ and $\overline{\tilde{g}}^{A}$ the four flavor $\pm \frac{1}{2}$ helicity gluinos, and $\phi_{A B}$ the six real 0 helicity scalar states. The Grassmann variables $\eta$ carry helicity $+\frac{1}{2}$ so that the whole multiplet carries helicity +1 .

We can write the amplitudes in the on-shell superspace with coordinates $\left(\lambda_{i}, \tilde{\lambda}_{i}, \eta_{i}\right)[18$, $23,54]$

$$
\mathcal{A}_{n}\left(\lambda_{i}, \tilde{\lambda}_{i}, \eta_{i}\right)=\mathcal{A}\left(\Phi_{1} \ldots \Phi_{n}\right) .
$$

Since the helicity of each supermultiplet $\Phi_{i}$ is 1 the amplitude obeys

$$
h_{i} \mathcal{A}_{n}\left(\lambda_{i}, \tilde{\lambda}_{i}, \eta_{i}\right)=\mathcal{A}_{n}\left(\lambda_{i}, \tilde{\lambda}_{i}, \eta_{i}\right)
$$

where $h_{i}$ is the helicity operator for the $i$ th leg. The component field amplitudes are then obtained by projecting upon the relevant terms in the $\eta_{i}$ expansion of the superamplitude via

$$
g_{i}^{+} \rightarrow \eta_{i}^{A}=0, \quad g_{i}^{-} \rightarrow \int d^{4} \eta_{i}=\int d \eta_{i}^{1} d \eta_{i}^{2} d \eta_{i}^{3} d \eta_{i}^{4}, \quad \tilde{g}_{i, A} \rightarrow \int d \eta^{A}, \quad \overline{\tilde{g}}_{i}^{A} \rightarrow-\int d^{4} \eta_{i} \eta_{i}^{A}
$$

Note that the super-amplitude $\mathcal{A}_{n}$ has a cyclic symmetry that can lead to many different but equivalent expressions in practice. In order to obtain compact expressions for component amplitudes often a judicious choice of this cyclic freedom can be made [29].

The general solution for tree super-amplitudes of Drummond and one of the present authors [29] takes the compact form

$$
\mathcal{A}_{n}^{\mathrm{N}^{p} \mathrm{MHV}}=\frac{\delta^{(4)}(p) \delta^{(8)}(q)}{\langle 12\rangle\langle 23\rangle \ldots\langle n 1\rangle} \sum_{\text {all paths of length } p} 1 \cdot R_{n, a_{1} b_{1}} \cdot R_{n,\left\{I_{2}\right\}, a_{2} b_{2}}^{\left.\left\{L_{2}\right\} ; U_{2}\right\}} \cdots \cdot R_{n,\left\{I_{p}\right\}, a_{p} b_{p}}^{\left\{L_{p}\right\} ;\left\{U_{p}\right\}},
$$

where $q^{\alpha A}=\sum_{i=1}^{n} \lambda_{i}^{\alpha} \eta_{i}^{A}$ is the total conserved fermionic momentum, and the dual superconformal $R$-invariant is

$$
R_{n ;\{I\} ; a b}=\tilde{R}_{n ;\{I\} ; a b} \delta^{(4)}\left(\sum_{i=1}^{n} \Xi_{n ;\{I\} ; a b}(i) \eta_{i}\right)
$$

in the notation of the previous sections. We now wish to project this result in on-shell superspace onto the relevant components for a general $g^{n-2 k}(q \bar{q})^{k}$ amplitude. For this purpose we set all of the $\eta_{i}$ associated to positive-helicity gluon states to zero. This leaves us with the $p+2+k$ remaining Grassmann numbers $\eta_{c_{i}}$ associated to the 'special' legs of helicities -1 and $\pm \frac{1}{2}$. To project onto a negative-gluon state at position $i$ one simply 
has to integrate eq. (7.5) against $\int d^{4} \eta_{i}$. Similarly, to project to a quark or anti-quark state at position $i$ of flavor $A_{i}$ one integrates eq. (7.5) against $\int d \eta_{i}^{A_{i}}$ or $-\int d^{4} \eta_{i} \eta_{i}^{A_{i}}$. All integrations have to be in color order.

In accord with our convention above the leg $n$ is chosen to be a negative-helicity gluon state, or an anti-quark if there are no negative-helicity gluons. This is a convenient choice because the only dependence of the super-amplitude on $\eta_{n}$ is through the total fermionic momentum conserving delta function, which can be written as

$$
\delta^{(8)}(q)=\delta^{(8)}\left(\sum_{i=0}^{p+k} \lambda_{c_{i}} \eta_{c_{i}}+\lambda_{n} \eta_{n}\right)=\delta^{(4)}\left(\sum_{i=1}^{p+k} \frac{\left\langle c_{0} c_{i}\right\rangle}{\left\langle c_{0} n\right\rangle} \eta_{c_{i}}+\eta_{n}\right) \delta^{(4)}\left(\sum_{i=0}^{p+k}\left\langle n c_{i}\right\rangle \eta_{c_{i}}\right) .
$$

For each path in eq. (7.5) the four-dimensional Grassmann delta functions in eq. (7.7), together with the $p$ delta functions arising from the $R$-invariants (7.6), may be written as

$$
\begin{aligned}
& \prod_{i=0}^{p+1} \delta^{(4)}\left(\sum_{j=0}^{p+k}\left(\Xi_{n}^{\text {path }}\right)_{i j} \eta_{c_{j}}\right):= \\
& \delta^{(4)}\left(\sum_{i=1}^{p+k} \frac{\left\langle c_{0} c_{i}\right\rangle}{\left\langle c_{0} n\right\rangle} \eta_{c_{i}}+\eta_{n}\right) \delta^{(4)}\left(\sum_{i=0}^{p+k}\left\langle n c_{i}\right\rangle \eta_{c_{i}}\right) \prod_{i=1}^{p} \delta^{(4)}\left(\sum_{j=0}^{p+k} \Xi_{n ;\left\{I_{i}\right\} ; a_{i} b_{i}}\left(c_{j}\right) \eta_{c_{j}}\right),
\end{aligned}
$$

with the $(p+2) \times(p+k+2)$ path-matrix $\Xi_{n}^{\text {path }}$. If we have a negative-helicity gluon at position $n$, the $\eta_{n}$ integration is trivial and we can drop the trial $\eta_{n}$ column and the row determined by the first delta function in eq. (7.8), ending up with the $(p+1) \times(p+k+1)$ path-matrix given in eq. (5.3). For the sake of readability we will drop the labels on the path-matrix in what follows, just denoting it by $\Xi$, and assume a negative-helicity gluon at position $n$. The projection to the general $g^{n-2 k}(q \bar{q})^{k}$ amplitude of eq. (4.2), with quarks of flavor $A_{i}$ at positions $c_{\alpha_{i}}$ and anti-quarks of flavor $B_{j}$ at positions $c_{\bar{\beta}_{j}}$,

$$
A_{(q \bar{q})^{k}, n}^{\mathrm{NPMHV}}\left(c_{0}, \ldots, c_{\alpha_{i}}^{A_{i}}, \ldots, c_{\bar{\beta}_{j}}^{B_{j}}, \ldots, c_{p+k}, n\right),
$$

is then performed via the Grassmann integrals

$$
(-1)^{k} \operatorname{sign}(\tau)\left(\prod_{\substack{j=0 \\ j \notin\left\{\alpha_{1}, \ldots, \alpha_{k}\right\}}}^{p+k} \int d^{4} \eta_{c_{j}}\right)\left(\prod_{l=1}^{k} \int d \eta_{c_{\alpha_{l}}}^{A_{l}} \eta_{c_{\bar{\beta}_{l}}}^{B_{l}}\right) \prod_{i=0}^{p+1} \delta^{(4)}\left(\sum_{j=0}^{p+k} \Xi_{i j} \eta_{c_{j}}\right) .
$$

Here $\operatorname{sign}(\tau)$ compensates the minus signs we encountered by permuting the quark and anti-quark Grassmann variables from color order to the canonical order $\prod_{l=1}^{k} \int d \eta_{c_{\alpha_{l}}}^{A_{l}} \eta_{c_{\bar{\beta}_{l}}}^{B_{l}}$.

Let us first consider the pure gluon case, i.e. $k=0$. Performing the change of variables $\eta_{c_{i}} \rightarrow \Xi_{i j}^{-1} \eta_{c_{j}}$ immediately gives

$$
\left(\prod_{j=0}^{p} \int d^{4} \eta_{c_{j}}\right) \prod_{i=0}^{p+1} \delta^{(4)}\left(\sum_{j=0}^{p} \Xi_{i j} \eta_{c_{j}}\right)=(\operatorname{det} \Xi)^{4}
$$


thereby proving eq. (4.2). To evaluate the general integral (7.9) we first perform the $(p+1)$ four-dimensional integrations with respect to the $\eta$ 's of the anti-quarks and gluons by making a change of variables similar to the pure-gluon case, leading to

$$
\operatorname{sign}(\tau)\left(\left.\operatorname{det} \Xi\right|_{q}\right)^{4} \prod_{l=1}^{k} \int d \eta_{c_{\alpha_{l}}}^{A_{l}} \sum_{\substack{i=0 \\ i \notin\left\{\alpha_{1}, \ldots, \alpha_{k}\right\}}}^{p+k}\left(\left.\Xi\right|_{q} ^{-1}\right)_{\bar{\beta}_{l} i} \sum_{j=1}^{k} \Xi_{i \alpha_{j}} \eta_{c_{\alpha_{j}}}^{B_{l}}
$$

Here $\left.\Xi\right|_{q}$ refers to the elimination of all quark columns in the path-matrix. We can simplify the sum over $i$ by making use of some basic facts of linear algebra. Namely, given a square matrix $M=\left(m_{i j}\right)$ with minors $M_{i j}$, its determinant and inverse can be written as

$$
\operatorname{det} M=\sum_{i}(-1)^{i+j} m_{i j} \operatorname{det} M_{i j} \quad \text { and } \quad\left(M^{-1}\right)_{i j}=(-1)^{i+j} \frac{\operatorname{det} M_{j i}}{\operatorname{det} M}
$$

Hence, eq. (7.11) simplifies to

$$
\operatorname{sign}(\tau)\left(\left.\operatorname{det} \Xi\right|_{q}\right)^{4-k} \prod_{l=1}^{k} \int d \eta_{c_{\alpha_{l}}}^{A_{l}} \sum_{j=1}^{k} \operatorname{det}\left(\left.\Xi\right|_{q}\left(\bar{\beta}_{l} \rightarrow \alpha_{j}\right)\right) \eta_{c_{\alpha_{j}}}^{B_{l}}
$$

where $\left.\Xi\right|_{q}\left(\bar{\beta}_{l} \rightarrow \alpha_{j}\right)$ denotes the replacement of an anti-quark column by a quark column. The remaining integrations are straightforward and give

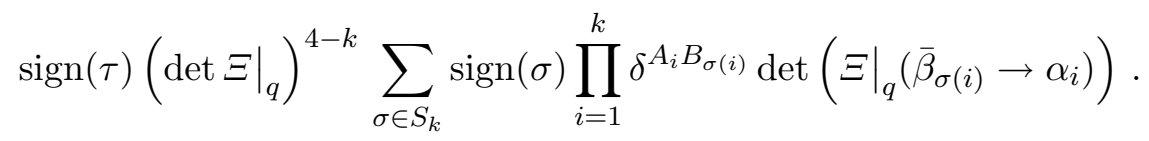

The general $\mathcal{N}=4$ super Yang-Mills $g^{n-2 k}(q \bar{q})^{k}$ amplitude is therefore

$$
\begin{aligned}
& A_{(q \bar{q})^{k}, n}^{\mathrm{NP} M H V}\left(c_{0}, \ldots, c_{\alpha_{i}}^{A_{i}}, \ldots, c_{\bar{\beta}_{j}}^{B_{j}} \ldots, c_{p+k}, n\right)=\frac{\delta^{(4)}(p) \operatorname{sign}(\tau)}{\langle 12\rangle\langle 23\rangle \ldots\langle n 1\rangle} \times \\
& \sum_{\substack{\text { all paths } \\
\text { of length } p}}\left(\prod_{i=1}^{p} \tilde{R}_{n ;\left\{I_{i}\right\} ; a_{i} b_{i}}^{L_{i} ; R_{i}}\right)\left(\left.\operatorname{det} \Xi\right|_{q}\right)^{4-k} \sum_{\sigma \in S_{k}} \operatorname{sign}(\sigma) \prod_{i=1}^{k} \delta^{A_{i} B_{\sigma(i)}} \operatorname{det}\left(\left.\Xi\right|_{q}\left(\bar{\beta}_{\sigma(i)} \rightarrow \alpha_{i}\right)\right) .
\end{aligned}
$$

Note that during the derivation of this formula we assumed that there is at least one negative-helicity gluon. The only change in the case $k=p+2$ is that the $\eta_{n}$ integration is no longer trivial and we put a gluino at position $n$. Hence, the path matrix has the size $(p+2) \times(2 p+4)$ and eq. (7.15) still holds as its derivation did not depend on the matrix 
dimensions. Using (7.8) the path matrix (4.12) then generalizes to the form

$$
\Xi:=\left(\begin{array}{llllll}
0 & \frac{\left\langle c_{0} c_{1}\right\rangle}{\left\langle c_{0} n\right\rangle} & \frac{\left\langle c_{0} c_{2}\right\rangle}{\left\langle c_{0} n\right\rangle} & \ldots & \frac{\left\langle c_{0} c_{p+k}\right\rangle}{\left\langle c_{0} n\right\rangle} & 1 \\
\left\langle n c_{0}\right\rangle & \left\langle n c_{1}\right\rangle & \left\langle n c_{2}\right\rangle & \ldots & \left\langle n c_{p+k}\right\rangle & 0 \\
\left(\Xi_{n}\right)_{a_{1} b_{1}}^{c_{0}} & \left(\Xi_{n}\right)_{a_{1} b_{1}}^{c_{1}} & \left(\Xi_{n}\right)_{a_{1} b_{1}}^{c_{2}} & \ldots\left(\Xi_{n}\right)_{a_{1} b_{1}}^{c_{p+k}} & 0 \\
\left(\Xi_{n}\right)_{\left\{I_{2}\right\} ; a_{2} b_{2}}^{c_{0}} & \left(\Xi_{n}\right)_{\left\{I_{2}\right\} ; a_{2} b_{2}}^{c_{1}} & \left(\Xi_{n}\right)_{\left\{I_{2}\right\} ; a_{2} b_{2}}^{c_{2}} & \ldots & \\
\vdots & \vdots & \left(\Xi_{n}\right)_{\left\{I_{2}\right\} ; a_{2} b_{2}}^{c_{p+k}} & 0 \\
\left(\Xi_{n}\right)_{\left\{I_{p}\right\} ; a_{p} b_{p}}^{c_{0}} & \left(\Xi_{n}\right)_{\left\{I_{p}\right\} ; a_{p} b_{p}}^{c_{1}} & \left(\Xi_{n}\right)_{\left\{I_{p}\right\} ; a_{p} b_{p}}^{c_{2}} \ldots\left(\Xi_{n}\right)_{\left\{I_{p}\right\} ; a_{p} b_{p}}^{c_{p}} & 0
\end{array}\right) .
$$

Generally the amplitudes take a more compact form if the gluino at position $n$ is taken to be of helicity $-1 / 2$. Several explicit formulas for the MHV and NMHV cases can be found in appendix B. In particular appendix B.2.3 discusses a case without a negative helicity gluon at position $n$.

As we are interested in QCD tree amplitudes, we need to decouple possible intermediate scalar states arising from the Yukawa couplings $\tilde{g}_{A} \tilde{g}_{B} \phi^{A B}$ in the $\mathcal{N}=4$ super Yang-Mills Lagrangian. As discussed in section 3, one case in which this can be achieved (although not the only one needed for QCD) is when the external fermion states all have the same flavor, due to the anti-symmetry of $\phi^{A B}=\epsilon^{A B C D} \phi_{C D}$. For this case, we specialize to $A_{i}=B_{i}=A$ for all external fermion legs $i$ in our master formula (7.15), and perform the sum over permutations explicitly, yielding

$$
\begin{aligned}
& A_{(q \bar{q})^{k}, n}^{\mathrm{NPMHV}}\left(c_{0}, \ldots, c_{\alpha_{i}}, \ldots, c_{\bar{\beta}_{j}} \ldots, c_{p+k}, n\right)= \\
& \left.\quad \frac{\delta^{(4)}(p) \operatorname{sign}(\tau)}{\langle 12\rangle\langle 23\rangle \ldots\langle n 1\rangle} \sum_{\begin{array}{c}
\text { all paths } \\
\text { of length } p
\end{array}}\left(\prod_{i=1}^{p} \tilde{R}_{n ;\left\{I_{i}\right\} ; a_{i} b_{i}}^{L_{i} ; R_{i}}\right) \operatorname{det} \Xi(q \leftrightarrow \bar{q})\right|_{\bar{q}}\left(\left.\operatorname{det} \Xi\right|_{q}\right)^{3},
\end{aligned}
$$

which reproduces eq. (5.2).

\section{Acknowledgments}

We thank Zvi Bern, Benedikt Biedermann, Jake Bourjaily, Harald Ita, Kemal Ozeren and Peter Uwer for helpful discussions. Several figures in this paper were made with JAXODRAW [55, 56], based on AXODRAW [57]. This work was supported by the Volkswagen Foundation, and by the US Department of Energy under contract DE-AC02-76SF00515.

\section{A Explicit formulae for gluon trees}

Here we explicitly apply our formula (4.2) to the NMHV and NNMHV cases. 


\section{A.1 NMHV amplitudes}

Without loss of generality, we take the negative-helicity gluons to be at positions $c_{0}, c_{1}, n$ with $c_{0}<c_{1}$. In the NMHV case only one path in figure 6 contributes and the pathmatrix is a $2 \times 2$ matrix whose determinant we denote by $D_{n, a_{1} b_{1}}^{c_{0} c_{1}}$. Hence, the NMHV gluon amplitude is given by

$$
A_{n}^{\mathrm{NMHV}}\left(c_{0}^{-}, c_{1}^{-}, n^{-}\right)=\frac{\delta^{(4)}(p)}{\langle 12\rangle \ldots\langle n 1\rangle} \sum_{2 \leq a_{1}<b_{1} \leq n-1} \tilde{R}_{n ; a_{1} b_{1}} \cdot\left(D_{n, a_{1} b_{1}}^{c_{0} c_{1}}\right)^{4}
$$

where the determinant of the path-matrix is given explicitly by

$$
D_{n, s t}^{a b}:=\left|\begin{array}{ll}
\langle n a\rangle & \langle n b\rangle \\
\left(\Xi_{n}\right)_{s t}^{a} & \left(\Xi_{n}\right)_{s t}^{b}
\end{array}\right| a \leq b \begin{cases}\langle n a\rangle\langle n t s \mid b\rangle & a<s \leq b<t, \\
\langle n a\rangle\langle b n\rangle x_{s t}^{2} & a<s<t \leq b, \\
\langle b a\rangle\langle n t s \mid n\rangle & s \leq a, b<t, \\
\langle n b\rangle\langle n s t \mid a\rangle & s \leq a<t \leq b .\end{cases}
$$

For $a>b$ one can use the antisymmetry of the determinant, $D_{n, s t}^{a b}=-D_{n, s t}^{b a}$. Equation (A.2) is exactly the result we already stated in eq. (2.7). This formula is implemented in GGT by GGTnmhvgluon.

\section{A.2 $\mathrm{N}^{2} \mathrm{MHV}$ amplitudes}

The negative-helicity gluons are taken to be $a^{-}, b^{-}, c^{-}, n^{-}$with $a<b<c$, without loss of generality. According to figure 6 there are two contributing paths. Denoting the determinants of their corresponding path-matrices by $D_{1}^{a b c}$ and $D_{2}^{a b c}$, the NNMHV gluon amplitude is given by

$$
\begin{array}{r}
A_{n}^{\mathrm{N}^{2} \mathrm{MHV}}\left(a^{-}, b^{-}, c^{-}, n^{-}\right)=\frac{\delta^{(4)}(p)}{\langle 12\rangle \ldots\langle n 1\rangle} \sum_{2 \leq a_{1}<b_{1}<n} \tilde{R}_{n ; a_{1} b_{1}} \cdot\left[\sum_{a_{1}+1 \leq a_{2}<b_{2} \leq b_{1}} \tilde{R}_{n ; b_{1} a_{1} ; a_{2} b_{2}}^{0 ; a_{1} b_{1}} \cdot\left(D_{1}^{a b c}\right)^{4}\right. \\
\left.+\sum_{b_{1} \leq a_{2}<b_{2}<n} \tilde{R}_{n ; a_{2} b_{2}}^{a_{1} b_{1} ; 0} \cdot\left(D_{2}^{a b c}\right)^{4}\right] .
\end{array}
$$

The explicit forms of the determinants of the path-matrices

$$
D_{1}^{a b c}\left(n, a_{1}, b_{1}, a_{2}, b_{2}\right):=\left|\begin{array}{lll}
\langle n a\rangle & \langle n b\rangle & \langle n c\rangle \\
\left(\Xi_{n}\right)_{a_{1} b_{1}}^{a} & \left(\Xi_{n}\right)_{a_{1} b_{1}}^{b} & \left(\Xi_{n}\right)_{a_{1} b_{1}}^{c} \\
\left(\Xi_{n}\right)_{b_{1}, a_{1} ; a_{2} b_{2}}^{a} & \left(\Xi_{n}\right)_{b_{1}, a_{1} ; a_{2} b_{2}}^{b} & \left(\Xi_{n}\right)_{b_{1}, a_{1} ; a_{2} b_{2}}^{c}
\end{array}\right|
$$

and

$$
D_{2}^{a b c}\left(n, a_{1}, b_{1}, a_{2}, b_{2}\right):=\left|\begin{array}{lll}
\langle n a\rangle & \langle n b\rangle & \langle n c\rangle \\
\left(\Xi_{n}\right)_{a_{1} b_{1}}^{a} & \left(\Xi_{n}\right)_{a_{1} b_{1}}^{b} & \left(\Xi_{n}\right)_{a_{1} b_{1}}^{c} \\
\left(\Xi_{n}\right)_{a_{2} b_{2}}^{a} & \left(\Xi_{n}\right)_{a_{2} b_{2}}^{b} & \left(\Xi_{n}\right)_{a_{2} b_{2}}^{c}
\end{array}\right|
$$


are given by

$$
D_{1}^{a b c}=\left\{\begin{array}{l}
\langle a n\rangle\left\langle n b_{1} a_{1} \mid b\right\rangle\left\langle n b_{1} a_{1} b_{2} a_{2} \mid c\right\rangle \\
\langle n a\rangle\left\langle n b_{1} a_{1} \mid b\right\rangle\left\langle n b_{1} a_{1} \mid c\right\rangle x_{a_{2} b_{2}}^{2} \\
\langle a n\rangle\langle b c\rangle\left\langle n b_{1} a_{1} a_{2} b_{2} \mid n b_{1} a_{1}\right\rangle \\
\langle a n\rangle\left\langle n b_{1} a_{1} \mid c\right\rangle\left\langle n b_{1} a_{1} a_{2} b_{2} \mid b\right\rangle \\
\langle n a\rangle\langle c n\rangle\left\langle n b_{1} a_{1} \mid b\right\rangle x_{a_{1} b_{1}}^{2} x_{a_{2} b_{2}}^{2} \\
\langle n a\rangle\langle n c\rangle x_{a_{1} b_{1}}^{2}\left\langle n b_{1} a_{1} a_{2} b_{2} \mid b\right\rangle \\
\langle a b\rangle\left\langle n b_{1} a_{1} \mid n\right\rangle\left\langle n b_{1} a_{1} b_{2} a_{2} \mid c\right\rangle \\
\langle c b\rangle\left\langle n b_{1} a_{1} \mid n\right\rangle\left\langle n b_{1} a_{1} b_{2} a_{2} \mid a\right\rangle \\
\langle b a\rangle\left\langle n b_{1} a_{1} \mid n\right\rangle\left\langle n b_{1} a_{1} \mid c\right\rangle x_{a_{2} b_{2}}^{2} \\
\left\langle n b_{1} a_{1} \mid n\right\rangle\left(x_{a_{2} b_{2}}^{2}\left\langle n b_{1} a_{1} \mid a\right\rangle\langle b c\rangle\right. \\
\left.\quad+\left\langle n b_{1} a_{1} a_{2} b_{2} \mid b\right\rangle\langle a c\rangle\right) \\
\langle a b\rangle\left\langle n b_{1} a_{1} \mid n\right\rangle\left\langle n b_{1} a_{1} a_{2} b_{2} \mid c\right\rangle \\
\langle c b\rangle\left\langle n b_{1} a_{1} \mid n\right\rangle\left\langle n b_{1} a_{1} a_{2} b_{2} \mid a\right\rangle \\
\langle b c\rangle\left\langle n b_{1} a_{1} \mid a\right\rangle\left\langle n b_{1} a_{1} \mid n\right\rangle x_{a_{2} b_{2}} \\
\langle c n\rangle\langle a b\rangle\left\langle n a_{1} \mid n b_{1}\right\rangle x_{a_{1} b_{1}}^{2} x_{a_{2} b_{2}}^{2} \\
\langle c n\rangle\left(x_{a_{2} b_{2}}^{2}\left\langle n b_{1} a_{1} \mid a\right\rangle\left\langle n a_{1} b_{1} \mid b\right\rangle\right. \\
\left.\quad+\left\langle n a_{1} b_{1} \mid a\right\rangle\left\langle n b_{1} a_{1} a_{2} b_{2} \mid b\right\rangle\right) \\
\langle n c\rangle\langle a b\rangle\left\langle n b_{1} a_{1} a_{2} b_{2} \mid n a_{1} b_{1}\right\rangle \\
\langle c n\rangle\left\langle n b_{1} a_{1} \mid a\right\rangle\left\langle n a_{1} b_{1} \mid b\right\rangle x_{a_{2} b_{2}}^{2} \\
\langle n c\rangle\left\langle n a_{1} b_{1} \mid b\right\rangle\left\langle n b_{1} a_{1} a_{2} b_{2} \mid a\right\rangle
\end{array}\right.
$$

$$
\begin{aligned}
& a<a_{1} \leq b, c<b_{1} \quad b<a_{2} \leq c<b_{2} \\
& a<a_{1} \leq b, c<b_{1} \quad b<a_{2}, b_{2} \leq c \\
& a<a_{1} \leq b, c<b_{1} \quad a_{1}<a_{2} \leq b, c<b_{2} \\
& a<a_{1} \leq b, c<b_{1} \quad a_{1}<a_{2} \leq b<b_{2} \leq c \\
& a<a_{1} \leq b<b_{1} \leq c \quad b<a_{2}, b_{2} \leq c \\
& a<a_{1} \leq b<b_{1} \leq c \quad a_{2} \leq b<b_{2} \\
& a_{1} \leq a, b, c<b_{1} \quad b<a_{2} \leq c<b_{2} \\
& a_{1} \leq a, b, c<b_{1} \quad a<a_{2} \leq b, c<b_{2} \\
& a_{1} \leq a, b, c<b_{1} \quad b<a_{2}, b_{2} \leq c \\
& a_{1} \leq a, b, c<b_{1} \quad a<a_{2} \leq b<b_{2} \leq c \\
& \\
& a_{1} \leq a, b, c<b_{1} \quad a_{2} \leq a, b<b_{2} \leq c \\
& a_{1} \leq a, b, c<b_{1} \quad a_{1}<a_{2} \leq a<b_{2} \leq b \\
& a_{1} \leq a, b, c<b_{1} \quad a_{1}<a_{2} \leq a<b_{2} \leq b \\
& a_{1} \leq a, b<b_{1} \leq c \quad b<a_{2}, b_{2} \leq b_{1} \\
& a_{1} \leq a, b<b_{1} \leq c \quad a<a_{2} \leq b<b_{2} \\
& \\
& a_{1} \leq a, b<b_{1} \leq c \quad a_{2} \leq a, b<b_{2} \\
& a_{1} \leq a, b<b_{1} \leq c \quad a<a_{2}, b_{2} \leq b \\
& a_{1} \leq a, b<b_{1} \leq c \quad a_{2} \leq a<b_{2} \leq b
\end{aligned}
$$

for $1<a_{1}<a_{2}<b_{2} \leq b_{1}<n$ and

$$
D_{2}^{a b c}= \begin{cases}\langle n a\rangle\left\langle n b_{1} a_{1} \mid b\right\rangle\left\langle n b_{2} a_{2} \mid c\right\rangle & a<a_{1} \leq b<b_{1} \leq c \quad a_{2} \leq c<b_{2} \\ \langle n a\rangle\langle c n\rangle\left\langle n b_{1} a_{1} \mid b\right\rangle x_{a_{2} b_{2}}^{2} & a<a_{1} \leq b<b_{1} \leq c \quad b_{2} \leq c \\ \langle n a\rangle\langle b n\rangle\left\langle n b_{2} a_{2} \mid c\right\rangle x_{a_{1} b_{1}}^{2} & a<a_{1}, b_{1} \leq b \quad b<a_{2} \leq c<b_{2} \\ \langle n a\rangle\langle b n\rangle\langle n c\rangle x_{a_{1} b_{1}}^{2} x_{a_{2} b_{2}}^{2} & a<a_{1}, b_{1} \leq b \quad b<a_{2}, b_{2} \leq c \\ \langle n a\rangle\langle b c\rangle\left\langle n b_{2} a_{2} \mid n\right\rangle x_{a_{1} b_{1}}^{2} & a<a_{1}, b_{1} \leq b \quad a_{2} \leq b, c<b_{2} \\ \langle n a\rangle\langle c n\rangle\left\langle n b_{2} a_{2} \mid b\right\rangle x_{a_{1} b_{1}}^{2} & a<a_{1}, b_{1} \leq b \quad a_{2} \leq b<b_{2} \leq c \\ \langle a b\rangle\left\langle n a_{1} b_{1} \mid n\right\rangle\left\langle n b_{2} a_{2} \mid c\right\rangle & a_{1} \leq a, b<b_{1} \leq c \quad a_{2} \leq c<b_{2} \\ \langle c n\rangle\langle a b\rangle\left\langle n a_{1} b_{1} \mid n\right\rangle x_{a_{2} b_{2}}^{2} & a_{1} \leq a, b<b_{1} \leq c \quad b_{2} \leq c \\ \langle n b\rangle\left\langle n a_{1} b_{1} \mid a\right\rangle\left\langle n b_{2} a_{2} \mid c\right\rangle & a_{1} \leq a<b_{1} \leq b \quad b<a_{2} \leq c<b_{2} \\ \langle n b\rangle\langle c n\rangle\left\langle n a_{1} b_{1} \mid a\right\rangle x_{a_{2} b_{2}}^{2} & a_{1} \leq a<b_{1} \leq b \quad b<a_{2}, b_{2} \leq c \\ \langle c b\rangle\left\langle n a_{1} b_{1} \mid a\right\rangle\left\langle n b_{2} a_{2} \mid n\right\rangle & a_{1} \leq a<b_{1} \leq b \quad a_{2} \leq b, c<b_{2} \\ \langle n c\rangle\left\langle n a_{1} b_{1} \mid a\right\rangle\left\langle n a_{2} b_{2} \mid b\right\rangle & a_{1} \leq a<b_{1} \leq b \quad a_{2} \leq b<b_{2} \leq c\end{cases}
$$


for $1<a_{1}<b_{1} \leq a_{2}<b_{2}<n$. For other orderings of $a, b, c$ one can use the total antisymmetry of $D_{1}^{a b c}$ and $D_{2}^{a b c}$ under permutations of $a, b, c$. It is quite astonishing that in 28 out of 30 cases these determinants are given by a single term. This formula is implemented in GGT by GGTnnmhvgluon.

\section{B Explicit formulae for trees with fermions}

Here we explicitly write out our formulas (5.2) and (6.2) for the MHV, NMHV and NNMHV cases with up to six fermions.

\section{B.1 MHV amplitudes}

The simplest amplitudes involving fermions are the MHV amplitudes. The amplitudes with one negative-helicity gluon $a$ and two fermions of opposite helicity and the same flavor, $b$ $\left(+\frac{1}{2}\right)$ and $\bar{c}\left(-\frac{1}{2}\right)$ (or vice-versa), are given by

$$
\begin{aligned}
& A_{n}\left(a^{-}, b, \bar{c}\right)=\delta^{(4)}(p) \frac{\langle a c\rangle^{3}\langle a b\rangle}{\langle 12\rangle \ldots\langle n 1\rangle}, \\
& A_{n}\left(a^{-}, \bar{b}, c\right)=-\delta^{(4)}(p) \frac{\langle a b\rangle^{3}\langle a c\rangle}{\langle 12\rangle \ldots\langle n 1\rangle} .
\end{aligned}
$$

These formulae correspond to case (1) in figure 3 . We note that the latter formula is related to the former one by a reflection symmetry, under which the cyclic ordering is reversed and there is a relabeling $b \leftrightarrow c$. In the NMHV case we will omit formulae that can be obtained from the presented formulae by a reflection symmetry.

An equally compact formula can be obtained for the MHV amplitudes with four fermions and only positive-helicity gluons:

$$
\begin{aligned}
& A_{n}\left(a^{A}, \bar{b}^{B}, c^{C}, \bar{d}^{D}\right)=\frac{\delta^{(4)}(p)\langle b d\rangle^{2}}{\langle 12\rangle \ldots\langle n 1\rangle}\left(\delta^{A B} \delta^{C D}\langle d a\rangle\langle c b\rangle-\delta^{A D} \delta^{B C}\langle d c\rangle\langle a b\rangle\right), \\
& A_{n}\left(a^{A}, b^{B}, \bar{c}^{C}, \bar{d}^{D}\right)=\frac{\delta^{(4)}(p)\langle c d\rangle^{2}}{\langle 12\rangle \ldots\langle n 1\rangle}\left(\delta^{A D} \delta^{B C}\langle d b\rangle\langle a c\rangle-\delta^{A C} \delta^{B D}\langle d a\rangle\langle b c\rangle\right),
\end{aligned}
$$

which in the single-flavor case simplifies to

$$
\begin{aligned}
& A_{n}(a, \bar{b}, c, \bar{d})=\frac{\delta^{(4)}(p)\langle b d\rangle^{3}\langle a c\rangle}{\langle 12\rangle \ldots\langle n 1\rangle}, \\
& A_{n}(a, b, \bar{c}, \bar{d})=-\frac{\delta^{(4)}(p)\langle c d\rangle^{3}\langle a b\rangle}{\langle 12\rangle \ldots\langle n 1\rangle} .
\end{aligned}
$$

Equation (B.6) corresponds to case (2a) in figure 3, whereas eq. (B.3) for $A=B \neq C=D$ corresponds to case $(2 \mathrm{~b})$.

To complete the list of MHV amplitudes with up to four fermions we also give the MHV amplitude with four positive-helicity fermions and one negative-helicity gluon:

$$
\begin{aligned}
A_{n}\left(a^{A}, b^{B}, c^{C}, d^{D}, n^{-}\right) & =\int d \eta_{a}^{A} \int d \eta_{b}^{B} \int d \eta_{c}^{C} \int d \eta_{d}^{D} \int d^{4} \eta_{n} \mathcal{A}_{n}^{\mathrm{M} H V} \\
& =\frac{\delta^{(4)}(p) \epsilon^{A B C D}}{\langle 12\rangle \ldots\langle n 1\rangle}\langle n a\rangle\langle n b\rangle\langle n c\rangle\langle n d\rangle .
\end{aligned}
$$

This amplitude is not needed for QCD. 


\section{B.2 NMHV amplitudes}

\section{B.2.1 Two fermions}

To illustrate the use of our master formula (5.2) we compute the NMHV amplitude with two opposite-helicity fermions at positions $a, \bar{b}$ and two negative-helicity gluons at positions $c$ and $n$. At this stage we leave the color order arbitrary. Starting with the path-matrix

$$
\Xi^{\text {path }}=\left(\begin{array}{lll}
\langle n c\rangle & \langle n a\rangle & \langle n \bar{b}\rangle \\
\left(\Xi_{n}\right)_{s t}^{c} & \left(\Xi_{n}\right)_{s t}^{a} & \left(\Xi_{n}\right)_{s t}^{\bar{b}}
\end{array}\right)
$$

we can immediately write down the amplitude

$$
\left(A_{n}\right)_{q \bar{q}}^{\mathrm{NMHV}}=\frac{\delta^{(4)}(p) \operatorname{sign}(\tau)}{\langle 12\rangle \ldots\langle n 1\rangle} \sum_{1<s<t<n} \tilde{R}_{n ; s t} D_{n ; s t}^{c a}\left(D_{n ; s t}^{c \bar{b}}\right)^{3}
$$

where the $2 \times 2$ determinant $D_{n ; s t}^{a b}$ has been defined in eq. (A.2). As already stated, the last equation holds for an arbitrary color ordering. In the following we take $a<b<c$ and specify the color ordering:

$$
\begin{aligned}
& A_{n}\left(a, b^{-}, \bar{c}, n^{-}\right)=\frac{\delta^{(4)}(p)}{\langle 12\rangle \ldots\langle n 1\rangle}\left[-\langle a b\rangle\langle b c\rangle^{3} \sum_{1<s \leq a, b, c<t<n}\langle n t s \mid n\rangle^{4} \tilde{R}_{n, s t}\right. \\
& -\langle b c\rangle^{3}\langle a n\rangle \sum_{a<s \leq b, c<t<n}\langle n t s \mid b\rangle\langle n t s \mid n\rangle^{3} \tilde{R}_{n, s t} \\
& -\langle c n\rangle^{3}\langle a n\rangle \sum_{a<s \leq b<t \leq c}\langle n s t \mid b\rangle^{3}\langle n t s \mid b\rangle \tilde{R}_{n, s t} \\
& \left.-\langle c n\rangle^{3}\langle a b\rangle \sum_{1<s \leq a, b<t \leq c}\langle n s t \mid b\rangle^{3}\langle n t s \mid n\rangle \tilde{R}_{n, s t}\right] \text {, } \\
& A_{n}\left(a, \bar{b}, c^{-}, n^{-}\right)=\frac{\delta^{(4)}(p)}{\langle 12\rangle \ldots\langle n 1\rangle}\left[+\langle a c\rangle\langle b c\rangle^{3} \sum_{1<s \leq a, b, c<t<n}\langle n t s \mid n\rangle^{4} \tilde{R}_{n, s t}\right. \\
& +\langle a n\rangle\langle b n\rangle^{3} \sum_{b<s \leq c<t<n}\langle n t s \mid c\rangle^{4} \tilde{R}_{n, s t} \\
& +\langle n c\rangle^{4}\langle a n\rangle\langle b n\rangle^{3} \sum_{b<s<t \leq c}\left(x_{s t}^{2}\right)^{4} \tilde{R}_{n, s t} \\
& +\langle c n\rangle^{4} \sum_{1<s \leq a, b<t \leq c}\langle n s t \mid b\rangle^{3}\langle n s t \mid a\rangle \tilde{R}_{n, s t} \\
& +\langle b c\rangle^{3}\langle a n\rangle \sum_{a<s \leq b, c<t<n}\langle n t s \mid c\rangle\langle n t s \mid n\rangle^{3} \tilde{R}_{n, s t} \\
& \left.+\langle c n\rangle^{4}\langle a n\rangle \sum_{a<s \leq b<t \leq c} x_{s t}^{2}\langle n s t \mid b\rangle^{3} \tilde{R}_{n, s t}\right]
\end{aligned}
$$

These simplified expressions are implemented in GGT by GGTnmhv2ferm (see appendix C). 


\section{B.2.2 Four fermions}

We proceed with the NMHV amplitude with four fermions at positions $a_{1}^{A_{1}}, a_{2}^{A_{2}}, \bar{b}_{1}^{B_{1}}, \bar{b}_{2}^{B_{2}}$ and one negative-helicity gluon. Without loss of generality we put the negative-helicity gluon at position $n$. Again we leave the color ordering arbitrary. A straightforward application of our formulas (6.2) and (5.2) yields

$$
\begin{aligned}
\left(A_{n}\right)_{(q \bar{q})^{2}}^{\mathrm{NMHV}}= & \frac{\delta^{(4)}(p) \operatorname{sign}(\tau)}{\langle 12\rangle \ldots\langle n 1\rangle} \times \\
& \times \sum_{1<s<t<n} \tilde{R}_{n ; s t}\left(D_{n ; s t}^{\bar{b}_{1} \bar{b}_{2}}\right)^{2}\left(\delta^{A_{1} B_{1}} \delta^{A_{2} B_{2}} D_{n ; s t}^{a_{1} \bar{b}_{2}} D_{n ; s t}^{\bar{b}_{1} a_{2}}-\delta^{A_{1} B_{2}} \delta^{A_{2} B_{1}} D_{n ; s t}^{a_{2} \bar{b}_{2}} D_{n ; s t}^{\bar{b}_{1} a_{1}}\right)
\end{aligned}
$$

in the $\mathcal{N}=4$ super Yang-Mills case, and

$$
\left(A_{n}\right)_{(q \bar{q})^{2}}^{\mathrm{NMHV}}=\frac{\delta^{(4)}(p) \operatorname{sign}(\tau)}{\langle 12\rangle \ldots\langle n 1\rangle} \sum_{1<s<t<n} \tilde{R}_{n ; s t}\left(D_{n ; s t}^{\bar{b}_{1} \bar{b}_{2}}\right)^{3} D_{n ; s t}^{a_{1} a_{2}}
$$

for single-flavor QCD, with $D_{n ; s t}^{a b}$ defined in equation (A.2). Taking $a<b<c<d$ we now specify the color ordering,

$$
\begin{aligned}
& A_{n}\left(a^{A}, b^{B}, \bar{c}^{C}, \bar{d}^{D}, n^{-}\right)=\frac{\delta^{(4)}(p)}{\langle 12\rangle \ldots\langle n 1\rangle} \times \\
& \times\left[+\delta^{A C} \delta^{B D}\langle a n\rangle\langle b c\rangle\langle c d\rangle^{2} \sum_{a<s \leq b, d<t<n}\langle n t s \mid d\rangle\langle n t s \mid n\rangle^{3} \tilde{R}_{n, s t}-(c \leftrightarrow d)\right. \\
& \quad+\delta^{A C} \delta^{B D}\langle a d\rangle\langle b c\rangle\langle d c\rangle^{2} \sum_{1<s \leq a, d<t<n}\langle n t s \mid n\rangle^{4} \tilde{R}_{n, s t}-(a \leftrightarrow b) \\
&+\delta^{A C} \delta^{B D}\langle n a\rangle\langle b c\rangle\langle d n\rangle^{3} \sum_{a<s \leq b, c<t \leq d} x_{s t}^{2}\langle n s t \mid n\rangle\langle n s t \mid c\rangle^{2} \tilde{R}_{n, s t} \\
& \quad-\delta^{A D} \delta^{B C}\langle a n\rangle\langle d n\rangle^{3} \sum_{a<s \leq b, c<t \leq d}\langle n t s \mid c\rangle\langle n s t \mid b\rangle\langle n s t \mid c\rangle^{2} \tilde{R}_{n, s t} \\
&\left.\quad+\delta^{A C} \delta^{B D}\langle b c\rangle\langle d n\rangle^{3} \sum_{1<s \leq a, c<t \leq d}\langle n s t \mid a\rangle\langle n t s \mid n\rangle\langle n s t \mid c\rangle^{2} \tilde{R}_{n, s t}-(a \leftrightarrow b)\right],
\end{aligned}
$$

where " $(c \leftrightarrow d)$ " implies the substitution $c \leftrightarrow d$ in the arguments of the spinor strings, as well as the corresponding substitution $C \leftrightarrow D$ in the arguments of the $\delta$ functions, but no change in the summation range. The other inequivalent orderings of quarks and 
anti-quarks are,

$$
\begin{aligned}
& A_{n}\left(a^{A}, \bar{b}^{B}, c^{C}, \bar{d}^{D}, n^{-}\right)=\frac{\delta^{(4)}(p)}{\langle 12\rangle \ldots\langle n 1\rangle} \times \\
& \times+\delta^{A B} \delta^{C D}\langle a n\rangle\langle b n\rangle^{3} \sum_{b<s \leq c, d<t<n}\langle n t s \mid c\rangle\langle n t s \mid d\rangle^{3} \tilde{R}_{n, s t} \\
&+\delta^{A B} \delta^{C D}\langle n a\rangle\langle c b\rangle\langle b d\rangle^{2} \sum_{a<s \leq b, d<t<n}\langle n t s \mid d\rangle\langle n t s \mid n\rangle^{3} \tilde{R}_{n, s t}-(b \leftrightarrow d) \\
&+\delta^{A B} \delta^{C D}\langle a d\rangle\langle b c\rangle\langle d b\rangle^{2} \sum_{1<s \leq a, d<t<n}\langle n t s \mid n\rangle^{4} \tilde{R}_{n, s t}-(a \leftrightarrow c) \\
&+\delta^{A B} \delta^{C D}\langle n a\rangle\langle d n\rangle^{3}\langle n b\rangle^{3} \sum_{b<s \leq c<t \leq d}\langle n t s \mid c\rangle\left(x_{s t}^{2}\right)^{3} \tilde{R}_{n, s t} \\
&+\delta^{A B} \delta^{C D}\langle n a\rangle\langle n c\rangle\langle n b\rangle^{3}\langle n d\rangle^{3} \sum_{b<s<t \leq c}\left(x_{s t}^{2}\right)^{4} \tilde{R}_{n, s t} \\
&+\delta^{A B} \delta^{C D}\langle n a\rangle\langle b c\rangle\langle d n\rangle^{3} \sum_{a<s \leq b, c<t \leq d} x_{s t}^{2}\langle n s t \mid n\rangle\langle n s t \mid b\rangle^{2} \tilde{R}_{n, s t} \\
&-\delta^{A D} \delta^{C B}\langle n a\rangle\langle d n\rangle^{3} \sum_{a<s \leq b, c<t \leq d}\langle n t s \mid b\rangle\langle n s t \mid c\rangle\langle n s t \mid b\rangle^{2} \tilde{R}_{n, s t} \\
&+\delta^{A B} \delta^{C D}\langle n a\rangle\langle n c\rangle\langle d n\rangle^{3} \sum_{a<s \leq b<t \leq c} x_{s t}^{2}\langle n s t \mid b\rangle^{3} \tilde{R}_{n, s t} \\
&+\delta^{A B} \delta^{C D}\langle b c\rangle\langle d n\rangle^{3} \sum_{1<s \leq a, c<t \leq d}\langle n s t \mid a\rangle\langle n t s \mid n\rangle\langle n s t \mid b\rangle^{2} \tilde{R}_{n, s t}-(a \leftrightarrow c) \\
&\left.\sum_{1<s \leq a, b<t \leq c}\langle n s t \mid a\rangle\langle n s t \mid b\rangle^{3} \tilde{R}_{n, s t}\right],
\end{aligned}
$$

$$
\begin{aligned}
A_{n}\left(a^{A}, \bar{b}^{B}, \bar{c}^{C}, d^{D},\right. & \left.n^{-}\right)=-\frac{\delta^{(4)}(p)}{\langle 12\rangle \ldots\langle n 1\rangle} \times \\
\times & +\delta^{A B} \delta^{C D}\langle a n\rangle\langle b n\rangle^{3} \sum_{b<s \leq c, d<t<n}\langle n t s \mid d\rangle\langle n t s \mid c\rangle^{3} \tilde{R}_{n, s t} \\
& +\delta^{A B} \delta^{C D}\langle n a\rangle\langle n d\rangle\langle b n\rangle^{3} \sum_{b<s \leq c<t \leq d} x_{s t}^{2}\langle n t s \mid c\rangle^{3} \tilde{R}_{n, s t} \\
& +\delta^{A B} \delta^{C D}\langle n a\rangle\langle d b\rangle\langle b c\rangle^{2} \sum_{a<s \leq b, d<t<n}\langle n t s \mid c\rangle\langle n t s \mid n\rangle^{3} \tilde{R}_{n, s t}-(b \leftrightarrow c) \\
& +\delta^{A B} \delta^{C D}\langle n a\rangle\langle n d\rangle\langle b c\rangle^{2} \sum_{a<s \leq b, c<t \leq d}\langle n t s \mid c\rangle\langle n s t \mid b\rangle\langle n t s \mid n\rangle^{2} \tilde{R}_{n, s t}-(b \leftrightarrow c) \\
& +\delta^{A B} \delta^{C D}\langle a c\rangle\langle b d\rangle\langle c b\rangle^{2} \sum_{1<s \leq a, d<t<n}\langle n t s \mid n\rangle^{4} \tilde{R}_{n, s t}-(a \leftrightarrow d)
\end{aligned}
$$




$$
\begin{aligned}
& +\delta^{A B} \delta^{C D}\langle a c\rangle\langle n d\rangle\langle c b\rangle^{2} \sum_{1<s \leq a, c<t \leq d}\langle n s t \mid b\rangle\langle n s t \mid n\rangle^{3} \tilde{R}_{n, s t}-(b \leftrightarrow c) \\
& +\delta^{A B} \delta^{C D}\langle n a\rangle\langle n d\rangle\langle n b\rangle^{3}\langle n c\rangle^{3} \sum_{b<s<t \leq c}\left(x_{s t}^{2}\right)^{4} \tilde{R}_{n, s t} \\
& +\delta^{A B} \delta^{C D}\langle n a\rangle\langle n d\rangle\langle c n\rangle^{3} \sum_{a<s \leq b<t \leq c} x_{s t}^{2}\langle n s t \mid b\rangle^{3} \tilde{R}_{n, s t} \\
& \left.+\delta^{A B} \delta^{C D}\langle n d\rangle\langle n c\rangle^{3} \sum_{1<s \leq a, b<t \leq c}\langle n s t \mid a\rangle\langle n s t \mid b\rangle^{3} \tilde{R}_{n, s t}\right]
\end{aligned}
$$

$$
\begin{aligned}
& A_{n}\left(\bar{a}^{A}, b^{B}, c^{C}, \bar{d}^{D}, n^{-}\right)=-\frac{\delta^{(4)}(p)}{\langle 12\rangle \ldots\langle n 1\rangle} \times \\
& \times[+\delta^{A B} \delta^{C D}\langle b n\rangle\langle a n\rangle^{3} \sum_{b<s \leq c, d<t<n}\langle n t s \mid c\rangle\langle n t s \mid d\rangle^{3} \tilde{R}_{n, s t} \\
&+\delta^{A B} \delta^{C D}\langle b d\rangle\langle a n\rangle^{3} \sum_{a<s \leq b, d<t<n}\langle n t s \mid n\rangle\langle n t s \mid c\rangle\langle n t s \mid d\rangle^{2} \tilde{R}_{n, s t}-(b \leftrightarrow c) \\
&+\delta^{A B} \delta^{C D}\langle b d\rangle\langle a c\rangle\langle d a\rangle^{2} \sum_{1<s \leq a, d<t<n}\langle n t s \mid n\rangle^{4} \tilde{R}_{n, s t}-(b \leftrightarrow c) \\
&+\delta^{A B} \delta^{C D}\langle n b\rangle\langle d n\rangle^{3}\langle n a\rangle^{3} \sum_{b<s \leq c<t \leq d}\langle n t s \mid c\rangle\left(x_{s t}^{2}\right)^{3} \tilde{R}_{n, s t} \\
&+\delta^{A B} \delta^{C D}\langle n b\rangle\langle n c\rangle\langle n a\rangle^{3}\langle n d\rangle^{3} \sum_{b<s<t \leq c}\left(x_{s t}^{2}\right)^{4} \tilde{R}_{n, s t} \\
&+\delta^{A B} \delta^{C D}\langle n a\rangle^{3}\langle n d\rangle^{3} \sum_{a<s \leq b, c<t \leq d}\langle n s t \mid b\rangle\langle n t s \mid c\rangle\left(x_{s t}^{2}\right)^{2} \tilde{R}_{n, s t}-(b \leftrightarrow c) \\
&+\delta^{A B} \delta^{C D}\langle n c\rangle\langle d n\rangle^{3}\langle n a\rangle^{3} \sum_{a<s \leq b<t \leq c}\langle n s t \mid b\rangle\left(x_{s t}^{2}\right)^{3} \tilde{R}_{n, s t} \\
&+\delta^{A B} \delta^{C D}\langle a c\rangle\langle d n\rangle^{3} \sum_{1<s \leq a, c<t \leq d}\langle n s t \mid b\rangle\langle n t s \mid n\rangle\langle n s t \mid a\rangle^{2} \tilde{R}_{n, s t}-(b \leftrightarrow c) \\
&\left.+\delta^{A B} \delta^{C D}\langle n c\rangle\langle n d\rangle^{3} \sum_{1<s \leq a, b<t \leq c}\langle n s t \mid b\rangle\langle n s t \mid a\rangle^{3} \tilde{R}_{n, s t}\right] .
\end{aligned}
$$


For $A \neq B$, and all fermions cyclically adjacent we have

$$
\begin{aligned}
& A_{n}\left(a^{A}, \overline{(a+1)}^{B},(a+2)^{B}, \overline{(a+3)}^{A}, n^{-}\right)=\frac{\delta^{(4)}(p)}{\langle 12\rangle \ldots\langle n 1\rangle} \times \\
& \quad\left[\langle a n\rangle\langle a+2 a+3\rangle\langle a+1 a+3\rangle^{2} \sum_{a+3<t<n}\langle n t a+2 \mid a+1\rangle\langle n t a+1 \mid n\rangle^{3} \tilde{R}_{n, a+1 t}\right. \\
& \quad+\langle a+2 a+3\rangle\langle a a+1\rangle\langle a+1 a+3\rangle^{2} \sum_{1<s \leq a, a+3<t<n}\langle n t s \mid n\rangle^{4} \tilde{R}_{n, s t} \\
& \quad+\langle a n\rangle\langle a+3 n\rangle^{3}\langle a+1 a+2\rangle^{4}\left\langle n\left|x_{n a+3}\right| a+2\right]\left\langle n\left|x_{n a+1}\right| a+1\right]\left\langle n\left|x_{n a+1}\right| a+2\right]^{2} \tilde{R}_{n, a+1 a+3} \\
& \left.\quad-\langle a a+1\rangle\langle a+3 n\rangle^{3} \sum_{1<s \leq a}\langle n s a+3 \mid a+2\rangle\langle n s a+3 \mid n\rangle\langle n s a+3 \mid a+1\rangle^{2} \tilde{R}_{n, s a+3}\right] .
\end{aligned}
$$

This amplitude may be used to generate the NMHV amplitudes for $V q \bar{q} g \ldots g$, as discussed in section 3 .

In the single-flavor case we obtain

$$
\begin{aligned}
& A_{n}\left(a, b, \bar{c}, \bar{d}, n^{-}\right)=\frac{\delta^{(4)}(p)}{\langle 12\rangle \ldots\langle n 1\rangle}\left[+\langle n a\rangle\langle c d\rangle^{3} \sum_{a<s \leq b, d<t<n}\langle n t s \mid b\rangle\langle n t s \mid n\rangle^{3} \tilde{R}_{n, s t}\right. \\
& +\langle a b\rangle\langle d c\rangle^{3} \sum_{1<s \leq a, d<t<n}\langle n t s \mid n\rangle^{4} \tilde{R}_{n, s t} \\
& +\langle n a\rangle\langle d n\rangle^{3} \sum_{a<s \leq b, c<t \leq d}\langle n t s \mid b\rangle\langle n s t \mid c\rangle^{3} \tilde{R}_{n, s t} \\
& \left.+\langle b a\rangle\langle d n\rangle^{3} \sum_{1<s \leq a, c<t \leq d}\langle n t s \mid n\rangle\langle n s t \mid c\rangle^{3} \tilde{R}_{n, s t}\right], \\
& A_{n}\left(a, \bar{b}, c, \bar{d}, n^{-}\right)=\frac{\delta^{(4)}(p)}{\langle 12\rangle \ldots\langle n 1\rangle}\left[+\langle a n\rangle\langle b n\rangle^{3} \sum_{b<s \leq c, d<t<n}\langle n t s \mid c\rangle\langle n t s \mid d\rangle^{3} \tilde{R}_{n, s t}\right. \\
& +\langle n a\rangle\langle d b\rangle^{3} \sum_{a<s \leq b, d<t<n}\langle n t s \mid c\rangle\langle n t s \mid n\rangle^{3} \tilde{R}_{n, s t} \\
& +\langle a c\rangle\langle b d\rangle^{3} \sum_{1<s \leq a, d<t<n}\langle n t s \mid n\rangle^{4} \tilde{R}_{n, s t} \\
& +\langle n a\rangle\langle d n\rangle^{3}\langle n b\rangle^{3} \sum_{b<s \leq c<t \leq d}\langle n t s \mid c\rangle\left(x_{s t}^{2}\right)^{3} \tilde{R}_{n, s t} \\
& +\langle n a\rangle\langle n c\rangle\langle n b\rangle^{3}\langle n d\rangle^{3} \sum_{b<s<t \leq c}\left(x_{s t}^{2}\right)^{4} \tilde{R}_{n, s t} \\
& +\langle n a\rangle\langle n d\rangle^{3} \sum_{a<s \leq b, c<t \leq d}\langle n t s \mid c\rangle\langle n s t \mid b\rangle^{3} \tilde{R}_{n, s t}
\end{aligned}
$$




$$
\begin{aligned}
& +\langle n a\rangle\langle n c\rangle\langle d n\rangle^{3} \sum_{a<s \leq b<t \leq c} x_{s t}^{2}\langle n s t \mid b\rangle^{3} \tilde{R}_{n, s t} \\
& +\langle a c\rangle\langle d n\rangle^{3} \sum_{1<s \leq a, c<t \leq d}\langle n t s \mid n\rangle\langle n s t \mid b\rangle^{3} \tilde{R}_{n, s t} \\
& \left.+\langle n c\rangle\langle n d\rangle^{3} \sum_{1<s \leq a, b<t \leq c}\langle n s t \mid a\rangle\langle n s t \mid b\rangle^{3} \tilde{R}_{n, s t}\right]
\end{aligned}
$$

$$
\begin{aligned}
A_{n}\left(a, \bar{b}, \bar{c}, d, n^{-}\right)=-\frac{\delta^{(4)}(p)}{\langle 12\rangle \ldots\langle n 1\rangle}[ & +\langle a n\rangle\langle b n\rangle^{3} \sum_{b<s \leq c, d<t<n}\langle n t s \mid d\rangle\langle n t s \mid c\rangle^{3} \tilde{R}_{n, s t} \\
& +\langle n a\rangle\langle n d\rangle\langle b n\rangle^{3} \sum_{b<s \leq c<t \leq d} x_{s t}^{2}\langle n t s \mid c\rangle^{3} \tilde{R}_{n, s t} \\
& +\langle n a\rangle\langle c b\rangle^{3} \sum_{a<s \leq b, d<t<n}\langle n t s \mid d\rangle\langle n t s \mid n\rangle^{3} \tilde{R}_{n, s t} \\
& +\langle n a\rangle\langle n d\rangle\langle b c\rangle^{3} \sum_{a<s \leq b, c<t \leq d} x_{s t}^{2}\langle n t s \mid n\rangle^{3} \tilde{R}_{n, s t} \\
& +\langle a d\rangle\langle b c\rangle^{3} \sum_{1<s \leq a, d<t<n}\langle n t s \mid n\rangle^{4} \tilde{R}_{n, s t} \\
& +\langle b c\rangle^{3}\langle n d\rangle \sum_{1<s \leq a, c<t \leq d}\langle n s t \mid a\rangle\langle n s t \mid n\rangle^{3} \tilde{R}_{n, s t} \\
& +\langle n a\rangle\langle n d\rangle\langle n b\rangle^{3}\langle n c\rangle^{3} \sum_{b<s<t \leq c}\left(x_{s t}^{2}\right)^{4} \tilde{R}_{n, s t} \\
& +\langle n a\rangle\langle n d\rangle\langle c n\rangle^{3} \sum_{a<s \leq b<t \leq c} x_{s t}^{2}\langle n s t \mid b\rangle^{3} \tilde{R}_{n, s t} \\
& \left.+\langle n d\rangle\langle n c\rangle^{3} \sum_{1<s \leq a, b<t \leq c}\langle n s t \mid a\rangle\langle n s t \mid b\rangle^{3} \tilde{R}_{n, s t}\right],
\end{aligned}
$$

$$
\begin{aligned}
A_{n}\left(\bar{a}, b, c, \bar{d}, n^{-}\right)=-\frac{\delta^{(4)}(p)}{\langle 12\rangle \ldots\langle n 1\rangle}[ & +\langle b n\rangle\langle a n\rangle^{3} \sum_{b<s \leq c, d<t<n}\langle n t s \mid c\rangle\langle n t s \mid d\rangle^{3} \tilde{R}_{n, s t} \\
& +\langle b c\rangle\langle a n\rangle^{3} \sum_{a<s \leq b, d<t<n}\langle n t s \mid n\rangle\langle n t s \mid d\rangle^{3} \tilde{R}_{n, s t} \\
& +\langle b c\rangle\langle a d\rangle^{3} \sum_{1<s \leq a, d<t<n}\langle n t s \mid n\rangle^{4} \tilde{R}_{n, s t} \\
& +\langle n b\rangle\langle d n\rangle^{3}\langle n a\rangle^{3} \sum_{b<s \leq c<t \leq d}\langle n t s \mid c\rangle\left(x_{s t}^{2}\right)^{3} \tilde{R}_{n, s t}
\end{aligned}
$$




$$
\begin{aligned}
& +\langle n b\rangle\langle n c\rangle\langle n a\rangle^{3}\langle n d\rangle^{3} \sum_{b<s<t \leq c}\left(x_{s t}^{2}\right)^{4} \tilde{R}_{n, s t} \\
& +\langle n a\rangle^{3}\langle n d\rangle^{3}\langle b c\rangle \sum_{a<s \leq b, c<t \leq d}\langle n t s \mid n\rangle\left(x_{s t}^{2}\right)^{3} \tilde{R}_{n, s t} \\
& +\langle n c\rangle\langle d n\rangle^{3}\langle n a\rangle^{3} \sum_{a<s \leq b<t \leq c}\langle n s t \mid b\rangle\left(x_{s t}^{2}\right)^{3} \tilde{R}_{n, s t} \\
& +\langle b c\rangle\langle d n\rangle^{3} \sum_{1<s \leq a, c<t \leq d}\langle n t s \mid n\rangle\langle n s t \mid a\rangle^{3} \tilde{R}_{n, s t} \\
& \left.+\langle n c\rangle\langle n d\rangle^{3} \sum_{1<s \leq a, b<t \leq c}\langle n s t \mid b\rangle\langle n s t \mid a\rangle^{3} \tilde{R}_{n, s t}\right] .
\end{aligned}
$$

These simplified expressions are implemented in GGT by GGTnmhv4fermS for the singleflavor case, and by GGTnmhv4ferm for the general-flavor case. See appendix C for the documentation.

\section{B.2.3 Six fermions}

In the case of the six-fermion NMHV amplitude there is no negative-helicity gluon for us to put at position $n$ as we did in the previous examples. The fermions are at positions $a_{1}^{A_{1}}, a_{2}^{A_{2}}, a_{3}^{A_{3}}, \bar{b}_{1}^{B_{1}}, \bar{b}_{2}^{B_{2}}$ and $\bar{n}^{B_{3}}$. This time the path-matrix (7.16) is given by

$$
\Xi^{\text {path }}=\left(\begin{array}{llllll}
\frac{\left\langle\bar{b}_{2} \bar{b}_{1}\right\rangle}{\left\langle\bar{b}_{2} n\right\rangle} & 0 & 1 \frac{\left\langle\bar{b}_{2} a_{1}\right\rangle}{\left\langle\bar{b}_{2} n\right\rangle} & \frac{\left\langle\bar{b}_{2} a_{2}\right\rangle}{\left\langle\bar{b}_{2} n\right\rangle} & \frac{\left\langle\bar{b}_{2} a_{3}\right\rangle}{\left\langle\bar{b}_{2} n\right\rangle} \\
\left\langle n \bar{b}_{1}\right\rangle & \left\langle n \bar{b}_{2}\right\rangle & 0 & \left\langle n a_{1}\right\rangle & \left\langle n a_{2}\right\rangle & \left\langle n a_{3}\right\rangle \\
\left(\Xi_{n}\right)_{s t} \bar{b}_{1} & \left(\Xi_{n}\right)_{s t} \bar{b}_{2} & 0 & \left(\Xi_{n}\right)_{s t}^{a_{1}} & \left(\Xi_{n}\right)_{s t}^{a_{2}} & \left(\Xi_{n}\right)_{s t}^{a_{3}}
\end{array}\right)
$$

As ingredients of formula (6.2) we need the determinants

$$
\begin{aligned}
\operatorname{det}\left(\left.\Xi^{\text {path }}\right|_{q}\right) & =D_{n ; s t}^{\bar{b}_{1} \bar{b}_{2},} \quad \operatorname{det}\left(\left.\Xi^{\text {path }}\right|_{q}\left(\bar{b}_{1} \rightarrow a_{i}\right)\right)=D_{n ; s t}^{a_{i} \bar{b}_{2}}, \\
\operatorname{det}\left(\left.\Xi^{\text {path }}\right|_{q}\left(\bar{b}_{2} \rightarrow a_{i}\right)\right) & =D_{n ; s t}^{\bar{b}_{1} a_{i}}, \quad \operatorname{det}\left(\left.\Xi^{\text {path }}\right|_{q}\left(\bar{n} \rightarrow a_{i}\right)\right)=D_{n ; s t}^{\bar{b}_{1} \bar{b}_{2} a_{i}} .
\end{aligned}
$$

We recall that $D_{n ; s t}^{a b}$ has been defined in eq. (A.2) and the $3 \times 3$ determinant $D_{n ; s t}^{a b c}$ reads

$$
D_{n ; s t}^{a b c}:=\left|\begin{array}{lll}
\frac{\langle b a\rangle}{\langle b n\rangle} & 0 & \frac{\langle b c\rangle}{\langle b n\rangle} \\
\langle n a\rangle & \langle n b\rangle & \langle n c\rangle \\
\left(\Xi_{n}\right)_{s t}^{a} & \left(\Xi_{n}\right)_{s t}^{b} & \left(\Xi_{n}\right)_{s t}^{c}
\end{array}\right|=\langle a b\rangle\left(\Xi_{n}\right)_{s t}^{c}+\langle b c\rangle\left(\Xi_{n}\right)_{s t}^{a}+\langle c a\rangle\left(\Xi_{n}\right)_{s t}^{b} .
$$


For $a<b<c$ we have

$$
D_{n ; s t}^{a b c}= \begin{cases}\langle a b\rangle\langle n t s \mid c\rangle & b<s \leq c<t \\ \langle a b\rangle\langle c n\rangle x_{s t}^{2} & b<s<t \leq c \\ \langle n t s \mid a\rangle\langle c b\rangle & a<s \leq b, c<t \\ \langle n a\rangle\langle b c\rangle x_{s t}^{2} & a<s<t \leq b \\ \langle a b\rangle\langle c n\rangle x_{s t}^{2}-\langle a c\rangle\langle n t s \mid b\rangle & a<s \leq b<t \leq c \\ \langle a b\rangle\langle n s t \mid c\rangle & s \leq a, b<t \leq c \\ \langle c b\rangle\langle n s t \mid a\rangle & s \leq a<t \leq b,\end{cases}
$$

and $D_{n ; s t}^{a b c}$ is totally antisymmetric in $a, b, c$. Thus, the $\mathcal{N}=4$ super Yang-Mills NMHV six-fermion amplitude is

$$
\begin{aligned}
\left(A_{n}\right)_{(q \bar{q})^{3}}^{\mathrm{NMHV}}=\frac{\delta^{(4)}(p) \operatorname{sign}(\tau)}{\langle 12\rangle \ldots\langle n 1\rangle} \sum_{1<s<t<n} \tilde{R}_{n ; s t} D_{n ; s t}^{\bar{b}_{1} \bar{b}_{2}}( & +\delta^{A_{1} B_{1}} \delta^{A_{2} B_{2}} \delta^{A_{3} B_{3}} D_{n ; s t}^{a_{1} \bar{b}_{2}} D_{n ; s t}^{\bar{b}_{1} a_{2}} D_{n ; s t}^{\bar{b}_{1} \bar{b}_{2} a_{3}} \\
& -\delta^{A_{1} B_{2}} \delta^{A_{2} B_{1}} \delta^{A_{3} B_{3}} D_{n ; s t}^{a_{2} \bar{b}_{2}} D_{n ; s t}^{\bar{b}_{1} a_{1}} D_{n ; s t}^{\bar{b}_{1} \bar{b}_{2} a_{3}} \\
& -\delta^{A_{1} B_{3}} \delta^{A_{2} B_{2}} \delta^{A_{3} B_{1}} D_{n ; s t}^{a_{3} \bar{b}_{2}} D_{n ; s t}^{\bar{b}_{1} a_{2}} D_{n ; s t}^{\bar{b}_{1} \bar{b}_{2} a_{1}} \\
& -\delta^{A_{1} B_{1}} \delta^{A_{2} B_{3}} \delta^{A_{3} B_{2}} D_{n ; s t}^{a_{1} \bar{b}_{2}} D_{n ; s t}^{\bar{b}_{1} a_{3}} D_{n ; s t}^{\bar{b}_{1} \bar{b}_{2} a_{2}} \\
& +\delta^{A_{1} B_{2}} \delta^{A_{2} B_{3}} \delta^{A_{3} B_{1}} D_{n ; s t}^{a_{3} \bar{b}_{2}} D_{n ; s t}^{\bar{b}_{1} a_{1}} D_{n ; s t}^{\bar{b}_{1} \bar{b}_{2} a_{2}} \\
& +\delta^{A_{1} B_{3}} \delta^{A_{2} B_{1}} \delta^{A_{3} B_{2}} D_{n ; s t}^{a_{2} \bar{b}_{2}} D_{n ; s t}^{\bar{b}_{1} a_{3}} D_{n ; s t}^{\bar{b}_{1} \bar{b}_{2} a_{1}}
\end{aligned}
$$

which in the single-flavor case (5.2) reduces to

$$
\left(A_{n}\right)_{(q \bar{q})^{3}}^{\mathrm{NMHV}}=\frac{\delta^{(4)}(p) \operatorname{sign}(\tau)}{\langle 12\rangle \ldots\langle n 1\rangle} \sum_{1<s<t<n} \tilde{R}_{n ; s t}\left(D_{n ; s t}^{\bar{b}_{1} \bar{b}_{2}}\right)^{3} D_{n ; s t}^{a_{1} a_{2} a_{3}} .
$$

These simplified expressions are implemented in GGT by the functions GGTnmhv6ferms for the single-flavor case and GGTnmhv6ferm for the general-flavor case. See appendix C for the documentation.

\section{B.3 N2 MHV amplitudes}

\section{B.3.1 Two fermions}

We continue the list of quark-gluon amplitudes by applying the master formulas (5.2) and (6.2) in the $\mathrm{N}^{2} \mathrm{MHV}$ case with up to six fermions. The amplitude with three negativehelicity gluons at positions $c_{1}, c_{2}, n$, a quark at position $a$ and an anti-quark at position $\bar{b}$, is

$$
\begin{aligned}
\left(A_{n}\right)_{(q \bar{q})}^{\mathrm{N}^{2} \mathrm{MHV}}=\frac{\delta^{(4)}(p) \operatorname{sign}(\tau)}{\langle 12\rangle \ldots\langle n 1\rangle} \sum_{2 \leq a_{1}<b_{1}<n} \tilde{R}_{n ; a_{1} b_{1}} \cdot & {\left[\sum_{a_{1}+1 \leq a_{2}<b_{2} \leq b_{1}} \tilde{R}_{n ; b_{1} a_{1} ; a_{2} b_{2}}^{0 ; a_{1} b_{1}} \cdot D_{1}^{c_{1} c_{2} a}\left(D_{1}^{c_{1} c_{2} \bar{b}}\right)^{3}\right.} \\
& \left.+\sum_{b_{1} \leq a_{2}<b_{2}<n} \tilde{R}_{n ; a_{2} b_{2}}^{a_{1} b_{1} ; 0} \cdot D_{2}^{c_{1} c_{2} a}\left(D_{2}^{c_{1} c_{2} \bar{b}}\right)^{3}\right]
\end{aligned}
$$

with the $3 \times 3$ determinants $D_{1}^{a b c}$ and $D_{2}^{a b c}$ from eqs. (A.6) and (A.7). 


\section{B.3.2 Four fermions}

For the amplitude with two negative-helicity gluons at positions $c, n$, as well as quarks and anti-quarks at positions $\alpha_{1}^{A_{1}}, \alpha_{2}^{A_{2}}$ and $\bar{\beta}_{1}^{B_{1}}, \bar{\beta}_{2}^{B_{2}}$, we obtain

$$
\begin{aligned}
& \left(A_{n}\right)_{(q \bar{q})^{2}}^{\mathrm{N}^{2} \mathrm{MHV}}=\frac{\delta^{(4)}(p) \operatorname{sign}(\tau)}{\langle 12\rangle \ldots\langle n 1\rangle} \sum_{2 \leq a_{1}<b_{1}<n} \tilde{R}_{n ; a_{1} b_{1}} \times \\
& \times\left[\sum_{a_{1}<a_{2}<b_{2} \leq b_{1}} \tilde{R}_{n ; b_{1} a_{1} ; a_{2} b_{2}}^{0 ; a_{1} b_{1}}\left(D_{1}^{c \bar{\beta}_{1} \bar{\beta}_{2}}\right)^{2}\left(\delta_{A_{1}}^{B_{1}} \delta_{A_{2}}^{B_{2}} D_{1}^{c \alpha_{1} \bar{\beta}_{2}} D_{1}^{c \bar{\beta}_{1} \alpha_{2}}-\delta_{A_{1}}^{B_{2}} \delta_{A_{2}}^{B_{1}} D_{1}^{c \alpha_{2} \bar{\beta}_{2}} D_{1}^{c \bar{\beta}_{1} \alpha_{1}}\right)\right. \\
& \left.\quad+\sum_{b_{1} \leq a_{2}<b_{2}<n} \tilde{R}_{n ; a_{2} b_{2}}^{a_{1} b_{1} ; 0}\left(D_{2}^{c \bar{\beta}_{1} \bar{\beta}_{2}}\right)^{2}\left(\delta_{A_{1}}^{B_{1}} \delta_{A_{2}}^{B_{2}} D_{2}^{c \alpha_{1} \bar{\beta}_{2}} D_{2}^{c \bar{\beta}_{1} \alpha_{2}}-\delta_{A_{1}}^{B_{2}} \delta_{A_{2}}^{B_{1}} D_{2}^{c \alpha_{2} \bar{\beta}_{2}} D_{2}^{c \bar{\beta}_{1} \alpha_{1}}\right)\right]
\end{aligned}
$$

in the $\mathcal{N}=4$ super Yang-Mills case, and

$$
\begin{array}{r}
\left(A_{n}\right)_{(q \bar{q})^{2}}^{\mathrm{N}^{2} \mathrm{MHV}}=\frac{\delta^{(4)}(p) \operatorname{sign}(\tau)}{\langle 12\rangle \ldots\langle n 1\rangle} \sum_{2 \leq a_{1}<b_{1}<n} \tilde{R}_{n ; a_{1} b_{1}} \cdot\left[\sum_{a_{1}<a_{2}<b_{2} \leq b_{1}} \tilde{R}_{n ; b_{1} a_{1} ; a_{2} b_{2}}^{0 ; a_{1} b_{1}} \cdot D_{1}^{c \alpha_{1} \alpha_{2}}\left(D_{1}^{c \bar{\beta}_{1} \bar{\beta}_{2}}\right)^{3}\right. \\
\left.+\sum_{b_{1} \leq a_{2}<b_{2}<n} \tilde{R}_{n ; a_{2} b_{2}}^{a_{1} b_{1} ; 0} \cdot D_{2}^{c_{1} c_{2} a}\left(D_{2}^{c_{1} c_{2} \bar{b}}\right)^{3}\right]
\end{array}
$$

for single-flavor QCD.

\section{B.3.3 Six fermions}

For the $\mathcal{N}=4$ super Yang-Mills amplitude with one negative-helicity gluon at position $n$, quarks and anti-quarks at positions $\alpha_{1}^{A_{1}}, \alpha_{2}^{A_{2}}, \alpha_{3}^{A_{3}}$ and $\bar{\beta}_{1}^{B_{1}}, \bar{\beta}_{2}^{B_{2}}, \bar{\beta}_{3}^{B_{3}}$, our master formula yields

$$
\begin{aligned}
& \left(A_{n}\right)_{(q \bar{q})^{3}}^{\mathrm{N}^{2} \mathrm{MHV}}=\frac{\delta^{(4)}(p) \operatorname{sign}(\tau)}{\langle 12\rangle \ldots\langle n 1\rangle} \sum_{2 \leq a_{1}<b_{1}<n} \tilde{R}_{n ; a_{1} b_{1} \times} \times \\
& \times\left[\sum_{a_{1}<a_{2}<b_{2} \leq b_{1}} \tilde{R}_{n b_{1} a_{1} ; a_{2} b_{2}}^{0 ; a_{1} b_{1}} D_{1}^{\bar{\beta}_{1} \bar{\beta}_{2} \bar{\beta}_{3}}\left(\delta_{A_{1}}^{B_{1}} \delta_{A_{2}}^{B_{2}} \delta_{A_{3}}^{B_{3}} D_{1}^{\alpha_{1} \bar{\beta}_{2} \bar{\beta}_{2}} D_{1}^{\bar{\beta}_{1} \alpha_{2} \bar{\beta}_{2}} D_{1}^{\bar{\beta}_{2} \bar{\beta}_{2} \alpha_{3}} \pm \text { perm. of }\left\{\begin{array}{l}
A_{i} \\
\alpha_{i}
\end{array}\right\}\right)\right. \\
& \left.\quad+\sum_{b_{1} \leq a_{2}<b_{2}<n} \tilde{R}_{n ; a_{2} b_{2}}^{a_{1} b_{1} ; 0} D_{2}^{\bar{\beta}_{1} \bar{\beta}_{2} \bar{\beta}_{3}}\left(\delta_{A_{1}}^{B_{1}} \delta_{A_{2}}^{B_{2}} \delta_{A_{3}}^{B_{3}} D_{2}^{\alpha_{1} \bar{\beta}_{2} \bar{\beta}_{2}} D_{2}^{\bar{\beta}_{1} \alpha_{2} \bar{\beta}_{2}} D_{2}^{\bar{\beta}_{2} \bar{\beta}_{2} \alpha_{3}} \pm \text { perm. of }\left\{\begin{array}{c}
A_{i} \\
\alpha_{i}
\end{array}\right\}\right)\right]
\end{aligned}
$$

which in the single-flavor case simplifies to

$$
\begin{aligned}
& \left(A_{n}\right)_{(q \bar{q})^{3}}^{\mathrm{N}^{2} \mathrm{MHV}}=\frac{\delta^{(4)}(p) \operatorname{sign}(\tau)}{\langle 12\rangle \ldots\langle n 1\rangle} \sum_{2 \leq a_{1}<b_{1}<n} \tilde{R}_{n ; a_{1} b_{1}} \cdot\left[\sum_{a_{1}<a_{2}<b_{2} \leq b_{1}} \tilde{R}_{n ; b_{1} a_{1} ; a_{2} b_{2}}^{0 ; a_{1} b_{1}} D_{1}^{\alpha_{1} \alpha_{2} \alpha_{3}}\left(D_{1}^{\bar{\beta}_{1} \bar{\beta}_{2} \bar{\beta}_{3}}\right)^{3}\right. \\
& \left.+\sum_{b_{1} \leq a_{2}<b_{2}<n} \tilde{R}_{n ; a_{2} b_{2}}^{a_{1} b_{1} ; 0} D_{2}^{\alpha_{1} \alpha_{2} \alpha_{3}}\left(D_{2}^{\bar{\beta}_{1} \bar{\beta}_{2} \bar{\beta}_{3}}\right)^{3}\right] \text {. }
\end{aligned}
$$


We recall that these formulas hold for arbitrary color-orderings of the $n$ partons.

We have implemented all of the above simplified expressions for NNMHV amplitudes with up to six fermions in the functions GGTnnmhv2ferm, GGTnnmhv4ferm, GGTnnmhv6ferm in the GGT package.

\section{The Mathematica package GGT}

Here we describe the Mathematica package GGT (gluon-gluino trees) provided with the arXiv.org submission of the present paper and also accessible via http://qft.physik. hu-berlin.de.

The idea is to provide the formulas derived in the present paper in computer-readable form, such that the interested reader can use them without having to type them in. We have also included a simple numerical evaluation routine for given phase-space points in the GGT package, as well as an interface to the spinor-helicity package S@M [58]. The issue of computer speed optimization will be commented upon below.

Let us now describe the different functions in GGT and then give a specific example. The following functions are provided in GGT

- GGTgluon $[\mathrm{n}, \mathrm{H}]$ gives the $n$-gluon amplitude (4.2), with the positions of the negative-helicity gluons given by the list $\mathrm{H}$.

- GGTfermionS [n, gluonlist, fermlist, afermlist] gives the $n$-parton amplitude (5.2) of an arbitrary number of gluons and singleflavor fermion/antifermions. The positions of the negative-helicity gluons, helicity $+\frac{1}{2}$ fermions, and helicity $-\frac{1}{2}$ anti-fermions are given by the lists gluonlist, fermlist, and afermlist, respectively.

- GGTfermion[n, gluonlist, fermlist, afermlist] is the generalization of GGTfermions to multiple fermion flavors, eq. (6.2). The positions of the negative-helicity gluons are given by the list gluonlist. The positions $q_{i}, \bar{q}_{i}$ and flavors $A_{i}, B_{i}$ of the helicity $+\frac{1}{2}$ fermions and helicity $-\frac{1}{2}$ anti-fermions are given by the lists fermlist $=\left\{\left\{q_{i}, A_{i}\right\}, \ldots\right\}$, and afermlist $=\left\{\left\{\bar{q}_{i}, A_{i}\right\}, \ldots\right\}$, respectively.

- GGTsuperamp $[n, k]$

is the $\mathrm{N}^{k} \mathrm{MHV}$ superamplitude of $n$ superfields, with the MHV superamplitude factored out, in terms of the $R$ invariants.

Let us give an example. We can load the GGT package using

$<$ GGT.m

Suppose we want to evaluate a gluon amplitude. Typing

GGTgluon $[6,\{3,5,6\}]$ 
prints the 6-gluon NMHV amplitude with helicity configuration ++-+-- ,

$$
\begin{aligned}
& \frac{1}{\langle 1 \mid 2\rangle\langle 2 \mid 3\rangle\langle 3 \mid 4\rangle\langle 4 \mid 5\rangle\langle 5 \mid 6\rangle\langle 6 \mid 1\rangle} \\
& \left(\frac{\langle 2 \mid 1\rangle\langle 4 \mid 3\rangle\left(s_{2,4}\langle 6 \mid 3\rangle\langle 6 \mid 5\rangle+\left\langle 6\left|x_{6,4}\right| x_{4,2} \mid 3\right\rangle\langle 6 \mid 5\rangle\right)^{4}}{s_{2,4}\left\langle 6\left|x_{6,2}\right| x_{2,4} \mid 3\right\rangle\left\langle 6\left|x_{6,2}\right| x_{2,4} \mid 4\right\rangle\left\langle 6\left|x_{6,4}\right| x_{4,2} \mid 1\right\rangle\left\langle 6\left|x_{6,4}\right| x_{4,2} \mid 2\right\rangle}\right. \\
& +\frac{\langle 2 \mid 1\rangle\langle 5 \mid 4\rangle\left(s_{2,5}\langle 6 \mid 3\rangle\langle 6 \mid 5\rangle\left\langle 6\left|x_{6,5}\right| x_{5,2} \mid 3\right\rangle\langle 6 \mid 5\rangle\right)^{4}}{s_{2,5}\left\langle 6\left|x_{6,2}\right| x_{2,5} \mid 4\right\rangle\left\langle 6\left|x_{6,2}\right| x_{2,5} \mid 5\right\rangle\left\langle 6\left|x_{6,5}\right| x_{5,2} \mid 1\right\rangle\left\langle 6\left|x_{6,5}\right| x_{5,2} \mid 2\right\rangle} \\
& \left.+\frac{\langle 3 \mid 2\rangle\langle 5 \mid 4\rangle\left(s_{3,5}\langle 6 \mid 3\rangle\langle 6 \mid 5\rangle+\left\langle 6\left|x_{6,5}\right| x_{5,3} \mid 3\right\rangle\langle 6 \mid 5\rangle\right)^{4}}{s_{3,5}\left\langle 6\left|x_{6,3}\right| x_{3,5} \mid 4\right\rangle\left\langle 6\left|x_{6,3}\right| x_{3,5} \mid 5\right\rangle\left\langle 6\left|x_{6,5}\right| x_{5,3} \mid 2\right\rangle\left\langle 6\left|x_{6,5}\right| x_{5,3} \mid 3\right\rangle}\right)
\end{aligned}
$$

GGT formatted the output for better readability. The underlying formula, which can be accessed explicitly, e.g. by using Inputform [...], depends on the following quantities: The spinor products $\langle i j\rangle$ are denoted by GGTspaa $[i, j]$. Differences between dual coordinates $x_{i, j}=p_{i}+p_{i+1}+\ldots+p_{j-1}$ are denoted by GGTx $[i, j]$. Finally, the abbreviation $x_{i j}^{2}=s_{i, j-1}$ is used and denoted by GGTs $[i, j-1]$.

In order to obtain numerical values, we can use the spinor-helicity package S@M [58]. The function GGTtoSpinors converts the expression into one that can be evaluated by the latter package. In our example, the commands

\section{$<$ Spinors.m}

GenMomenta $[1,2,3,4,5,6]$

load the S@M package and use one of its functions to generate arbitrary momenta for a sixparticle scattering process. Finally, numerical values of the amplitude at that phase-space point can be obtained by the command

GGTtoSpinors [GGTgluon $[6,\{3,5,6\}]] / / \mathrm{N}$

A faster implementation for the numerical evaluation of the GGT formulas is provided by the function GGTgenvar [P] which generates the spinors and region momenta for a numerical evaluation of an amplitude at a desired phase-space point $P=\left\{p_{1}, p_{2}, \ldots, p_{n}\right\}$. For example, for the kinematic point given in eq. (4.6) of ref. [59] (which to save space we give here to only three significant digits), one would use

GGTgenvar $[\{\{-3.0,2.12,1.06,1.84\},\{-3.0,-2.12,-1.06,-1.84\},\{2.0,2.0,0.0,0.0\}$, $\{0.857,-0.316,0.797,0.0\},\{1.0,-0.184,0.465,0.866\},\{2.14,-1.5,-1.26,-0.866\}\}]$

One can then evaluate an amplitude numerically by the command

GGTnumeric [GGTfermions $[6,\{1,6\},\{2,4\},\{3,5\}]]$

$-0.496838+0.0714737 i$

This approach is considerably faster than the GGTtoSpinors $[\ldots] / / \mathrm{N}$ function discussed above.

Let us comment about the evaluation time needed using our approach. It is clear that for any serious applications or for comparisons with other methods, one should implement our analytical formulas using a low-level programming language, such as $\mathrm{C}, \mathrm{C}++$ 
or FORTRAN. For example, an implementation of the NMHV formulas in C++ results in a speedup of orders of magnitude over a similar implementation in Mathematica. Moreover, it is important to efficiently cache (store the numerical values of) quantities that are used repeatedly. In this spirit, the Mathematica demonstration package GGT provides a computer-readable version of the formulas needed for such an approach, so that the user does not have to type them in manually.

Our analytical formulas are very similar, and in some cases identical, to the ones obtained in a very recent paper [60]. The latter also correspond to solutions of the BCFW recursion relations, based on refs. [61, 62], but may differ in form since they can correspond to different factorization channels. Another difference is that they are written using momentum-twistor variables [63, 64]. Ref. [60] contains a numerical Mathematica implementation of these formulas. When the formulas of our paper and that of ref. [60] are both implemented with appropriate caching in $\mathrm{C}++$, for the NMHV tree amplitudes for $V q \bar{q} g g g g g$ and $V q \bar{q} Q \bar{Q} g g g$, their evaluation time is similar [65].

We remark that in approaches based on BCFW recursion relations, the asymptotic number of terms in $\mathrm{N}^{k} \mathrm{MHV}_{n}$ amplitudes as $n$ becomes large is quadratic in $n$ for NMHV, quartic for NNMHV and worse for higher $k$. This is the reason we especially simplified the NMHV and NNMHV formulas presented in our paper, since we expect that they will be the most useful for practical applications, especially for small $n$. For $k>2$ and large $n$ there are at least two efficient numerical strategies making use of these formulae. First, one could use our formulae as initial conditions for a numerical implementation of the BCFW recursion relations, as described in section 3. Alternatively, one could use the BerendsGiele approach for $k>2$, implemented using an efficient caching, in combination with our formulas for $k \leq 2$ [66].

We also included further functions that evaluate directly the simplified amplitudes of appendix A and B. They can be accessed via the following functions.

- GGTnmhvgluon $[n, a, b]$

is the simplified $n$-parton NMHV gluon amplitude with negative-helicity gluons at positions $a, b$ and $n$.

- GGTnnmhvgluon $[n, a, b, c]$

is the simplified $n$-parton NNMHV gluon amplitude with negative-helicity gluons at positions $a, b, c$ and $n$.

- GGTnmhv2ferm $[n, c, a, \bar{b}]$

is the simplified $n$-parton NMHV two-fermion amplitude with negative-helicity gluons at positions $c, n$ and a fermion/anti-fermion at positions $a$ and $\bar{b}$.

- GGTnnmhv2ferm $\left[n, c_{1}, c_{2}, a, \bar{b}\right]$

is the simplified $n$-parton NNMHV two-fermion amplitude with negative-helicity gluons at positions $c_{1}, c_{2}$ and $n$ and a fermion/anti-fermion at position $a$ and $\bar{b}$.

- GGTnmhv4ferm $\left[n,\left\{\left\{a_{1}, A_{1}\right\},\left\{a_{2}, A_{2}\right\}\right\},\left\{\left\{\bar{b}_{1}, B_{1}\right\},\left\{\bar{b}_{2}, B_{2}\right\}\right\}\right]$

is the simplified $n$-parton NMHV four-fermion amplitude with a negative-helicity 
gluon at position $n$, two gluinos of flavors $A_{i}$ at positions $a_{i}$ and two anti-gluinos of flavors $B_{i}$ at positions $\bar{b}_{i}$.

- GGTnmhv4fermS $\left[n,\left\{a_{1}, a_{2}\right\},\left\{\bar{b}_{1}, \bar{b}_{2}\right\}\right]$

is the simplified $n$-parton NMHV four-fermion amplitude with a negative-helicity gluon at position $n$ and equally flavored gluinos/anti-gluinos at positions $a_{i}$ and $\bar{b}_{i}$, respectively.

- GGTnnmhv4ferm $\left[n, c,\left\{\left\{a_{1}, A_{1}\right\},\left\{a_{2}, A_{2}\right\}\right\},\left\{\left\{\bar{b}_{1}, B_{1}\right\},\left\{\bar{b}_{2}, B_{2}\right\}\right\}\right]$

is the simplified $n$-parton NNMHV four-fermion amplitude with two negative-helicity gluons at position $c, n$, two gluinos of flavors $A_{i}$ at positions $a_{i}$ and two anti-gluinos of flavors $B_{i}$ at positions $\bar{b}_{i}$.

- GGTnnmhv4fermS $\left[n, c,\left\{a_{1}, a_{2}\right\},\left\{\bar{b}_{1}, \bar{b}_{2}\right\}\right]$

is the simplified $n$-parton NNMHV four-fermion amplitude with negative-helicity gluons at positions $c, n$ and equally flavored gluinos/anti-gluinos at positions $a_{i}$ and $\bar{b}_{i}$, respectively.

- GGTnmhv6ferm $\left[n, B_{3},\left\{\left\{a_{1}, A_{1}\right\},\left\{a_{2}, A_{2}\right\},\left\{a_{3}, A_{3}\right\}\right\},\left\{\left\{\bar{b}_{1}, B_{1}\right\},\left\{\bar{b}_{2}, B_{2}\right\}\right\}\right]$ is the simplified $n$-parton NMHV six-fermion amplitude with three gluinos of flavors $A_{i}$ at positions $a_{i}$ and three anti-gluinos of flavors $B_{i}$ at positions $\bar{b}_{i}$. Note that $\bar{b}_{3}=n$.

- GGTnmhv6ferms $\left[n,\left\{a_{1}, a_{2}, a_{3}\right\},\left\{\bar{b}_{1}, \bar{b}_{2}\right\}\right]$

is the simplified $n$-parton NMHV six-fermion amplitude with equally flavored gluinos/ anti-gluinos at positions $a_{i}$ and $\bar{b}_{i}$, respectively. Note that $\bar{b}_{3}=n$.

- GGTnnmhv6ferm $\left[n,\left\{\left\{a_{1}, A_{1}\right\},\left\{a_{2}, A_{2}\right\},\left\{a_{3}, A_{3}\right\}\right\},\left\{\left\{\bar{b}_{1}, B_{1}\right\},\left\{\bar{b}_{2}, B_{2}\right\},\left\{\bar{b}_{3}, B_{3}\right\}\right\}\right]$ is the simplified $n$-parton NNMHV six-fermion amplitude with a negative helicity gluon at position $n$ and three gluinos of flavors $A_{i}$ at positions $a_{i}$ and three antigluinos of flavors $B_{i}$ at positions $\bar{b}_{i}$.

- GGTnnmhv6fermS $\left[n,\left\{a_{1}, a_{2}, a_{3}\right\},\left\{\bar{b}_{1}, \bar{b}_{2}, \bar{b}_{3}\right\}\right]$

is the simplified $n$-parton NNMHV six-fermion amplitude with a negative helicity gluon at position $n$ and equally flavored gluinos/anti-gluinos at positions $a_{i}$ and $\bar{b}_{i}$, respectively.

The full list of functions available in GGT can be obtained by typing

\section{\$GGTfunctions}

along with the documentation of each implemented function that can be accessed via the command

?GGTgluon

for example. 
Open Access. This article is distributed under the terms of the Creative Commons Attribution Noncommercial License which permits any noncommercial use, distribution, and reproduction in any medium, provided the original author(s) and source are credited.

\section{References}

[1] T. Stelzer and W.F. Long, Automatic generation of tree level helicity amplitudes, Comput. Phys. Commun. 81 (1994) 357 [hep-ph/9401258] [SPIRES].

[2] J. Alwall et al., MadGraph/MadEvent v4: The New Web Generation, JHEP 09 (2007) 028 [arXiv:0706.2334] [SPIRES].

[3] A. Pukhov et al., CompHEP: A package for evaluation of Feynman diagrams and integration over multi-particle phase space. User's manual for version 33, hep-ph/9908288 [SPIRES].

[4] F. Krauss, R. Kuhn and G. Soff, AMEGIC ++ 1.0: A Matrix element generator in $\mathrm{C}++$, JHEP 02 (2002) 044 [hep-ph/0109036] [SPIRES].

[5] F.A. Berends and W.T. Giele, Recursive Calculations for Processes with n Gluons, Nucl. Phys. B 306 (1988) 759 [SPIRES].

[6] T. Gleisberg and S. Hoeche, Comix, a new matrix element generator, JHEP 12 (2008) 039 [arXiv: 0808.3674] [SPIRES].

[7] F. Caravaglios and M. Moretti, An algorithm to compute Born scattering amplitudes without Feynman graphs, Phys. Lett. B 358 (1995) 332 [hep-ph/9507237] [SPIRES].

[8] F. Caravaglios, M.L. Mangano, M. Moretti and R. Pittau, A new approach to multi-jet calculations in hadron collisions, Nucl. Phys. B 539 (1999) 215 [hep-ph/9807570] [SPIRES].

[9] A. Kanaki and C.G. Papadopoulos, HELAC: A package to compute electroweak helicity amplitudes, Comput. Phys. Commun. 132 (2000) 306 [hep-ph/0002082] [SPIRES].

[10] A. Cafarella, C.G. Papadopoulos and M. Worek, Helac-Phegas: a generator for all parton level processes, Comput. Phys. Commun. 180 (2009) 1941 [arXiv:0710.2427] [SPIRES].

[11] M. Moretti, T. Ohl and J. Reuter, O'Mega: An optimizing matrix element generator, hep-ph/0102195 [SPIRES].

[12] W. Kilian, T. Ohl and J. Reuter, WHIZARD: Simulating Multi-Particle Processes at LHC and ILC, arXiv:0708.4233 [SPIRES].

[13] S.J. Parke and T.R. Taylor, Perturbative QCD Utilizing Extended Supersymmetry, Phys. Lett. B 157 (1985) 81 [SPIRES].

[14] Z. Kunszt, Combined Use of the Calkul Method and $N=1$ Supersymmetry to Calculate QCD Six Parton Processes, Nucl. Phys. B 271 (1986) 333 [SPIRES].

[15] M.T. Grisaru, H.N. Pendleton and P. van Nieuwenhuizen, Supergravity and the S Matrix, Phys. Rev. D 15 (1977) 996 [SPIRES].

[16] M.T. Grisaru and H.N. Pendleton, Some Properties of Scattering Amplitudes in Supersymmetric Theories, Nucl. Phys. B 124 (1977) 81 [SPIRES].

[17] S.J. Parke and T.R. Taylor, An Amplitude for n Gluon Scattering, Phys. Rev. Lett. 56 (1986) 2459 [SPIRES].

[18] V.P. Nair, A current algebra for some gauge theory amplitudes, Phys. Lett. B 214 (1988) 215 [SPIRES]. 
[19] Z. Bern, L.J. Dixon, D.C. Dunbar and D.A. Kosower, One-Loop n-Point Gauge Theory Amplitudes, Unitarity and Collinear Limits, Nucl. Phys. B 425 (1994) 217 [hep-ph/9403226] [SPIRES].

[20] Z. Bern, L.J. Dixon, D.C. Dunbar and D.A. Kosower, Fusing gauge theory tree amplitudes into loop amplitudes, Nucl. Phys. B 435 (1995) 59 [hep-ph/9409265] [SPIRES].

[21] Z. Bern, L.J. Dixon and D.A. Kosower, On-Shell Methods in Perturbative QCD, Annals Phys. 322 (2007) 1587 [arXiv:0704.2798] [SPIRES].

[22] C.F. Berger and D. Forde, Multi-Parton Scattering Amplitudes via On-Shell Methods, arXiv:0912.3534 [SPIRES].

[23] E. Witten, Perturbative gauge theory as a string theory in twistor space, Commun. Math. Phys. 252 (2004) 189 [hep-th/0312171] [SPIRES].

[24] R. Britto, F. Cachazo and B. Feng, New Recursion Relations for Tree Amplitudes of Gluons, Nucl. Phys. B 715 (2005) 499 [hep-th/0412308] [SPIRES].

[25] R. Britto, F. Cachazo, B. Feng and E. Witten, Direct Proof Of Tree-Level Recursion Relation In Yang-Mills Theory, Phys. Rev. Lett. 94 (2005) 181602 [hep-th/0501052] [SPIRES].

[26] R. Britto, B. Feng, R. Roiban, M. Spradlin and A. Volovich, All split helicity tree-level gluon amplitudes, Phys. Rev. D 71 (2005) 105017 [hep-th/0503198] [SPIRES].

[27] N. Arkani-Hamed, F. Cachazo, C. Cheung and J. Kaplan, A Duality For The S Matrix, JHEP 03 (2010) 020 [arXiv:0907.5418] [SPIRES].

[28] M. Bianchi, H. Elvang and D.Z. Freedman, Generating Tree Amplitudes in $N=4 S Y M$ and $N=8$ SG, JHEP 09 (2008) 063 [arXiv:0805.0757] [SPIRES].

[29] J.M. Drummond and J.M. Henn, All tree-level amplitudes in N=4 SYM, JHEP 04 (2009) 018 [arXiv:0808.2475] [SPIRES].

[30] J.M. Drummond, J. Henn, G.P. Korchemsky and E. Sokatchev, Dual superconformal symmetry of scattering amplitudes in $N=4$ super-Yang-Mills theory, Nucl. Phys. B 828 (2010) 317 [arXiv:0807.1095] [SPIRES].

[31] A. Brandhuber, P. Heslop and G. Travaglini, A note on dual superconformal symmetry of the $N=4$ super Yang-Mills S-matrix, Phys. Rev. D 78 (2008) 125005 [arXiv:0807.4097] [SPIRES].

[32] J.M. Drummond, J. Henn, G.P. Korchemsky and E. Sokatchev, Generalized unitarity for $N=4$ super-amplitudes, arXiv:0808.0491 [SPIRES].

[33] J.M. Drummond, J.M. Henn and J. Plefka, Yangian symmetry of scattering amplitudes in $N=4$ super Yang-Mills theory, JHEP 05 (2009) 046 [arXiv:0902.2987] [SPIRES].

[34] J.M. Drummond, Hidden Simplicity of Gauge Theory Amplitudes, Class. Quant. Grav. 27 (2010) 214001 [arXiv:1010.2418] [SPIRES].

[35] C.F. Berger et al., Precise Predictions for $W+4$ Jet Production at the Large Hadron Collider, arXiv:1009.2338 [SPIRES].

[36] G. Ossola, C.G. Papadopoulos and R. Pittau, Reducing full one-loop amplitudes to scalar integrals at the integrand level, Nucl. Phys. B 763 (2007) 147 [hep-ph/0609007] [SPIRES].

[37] G. Ossola, C.G. Papadopoulos and R. Pittau, CutTools: a program implementing the OPP reduction method to compute one-loop amplitudes, JHEP 03 (2008) 042 [arXiv:0711.3596] [SPIRES]. 
[38] G. Ossola, C.G. Papadopoulos and R. Pittau, On the Rational Terms of the one-loop amplitudes, JHEP 05 (2008) 004 [arXiv:0802.1876] [SPIRES].

[39] R.K. Ellis, W.T. Giele and Z. Kunszt, A Numerical Unitarity Formalism for Evaluating One-Loop Amplitudes, JHEP 03 (2008) 003 [arXiv:0708.2398] [SPIRES].

[40] W.T. Giele, Z. Kunszt and K. Melnikov, Full one-loop amplitudes from tree amplitudes, JHEP 04 (2008) 049 [arXiv: 0801.2237] [SPIRES].

[41] W.T. Giele and G. Zanderighi, On the Numerical Evaluation of One-Loop Amplitudes: The Gluonic Case, JHEP 06 (2008) 038 [arXiv:0805.2152] [SPIRES].

[42] C.F. Berger et al., An Automated Implementation of On-Shell Methods for One-Loop Amplitudes, Phys. Rev. D 78 (2008) 036003 [arXiv:0803.4180] [SPIRES].

[43] Z. Bern and A.G. Morgan, Massive Loop Amplitudes from Unitarity, Nucl. Phys. B 467 (1996) 479 [hep-ph/9511336] [SPIRES].

[44] Z. Bern, L.J. Dixon, D.C. Dunbar and D.A. Kosower, One-loop self-dual and $N=4$ super Yang-Mills, Phys. Lett. B 394 (1997) 105 [hep-th/9611127] [SPIRES].

[45] C. Anastasiou, R. Britto, B. Feng, Z. Kunszt and P. Mastrolia, D-dimensional unitarity cut method, Phys. Lett. B 645 (2007) 213 [hep-ph/0609191] [SPIRES].

[46] R. Britto and B. Feng, Integral Coefficients for One-Loop Amplitudes, JHEP 02 (2008) 095 [arXiv:0711.4284] [SPIRES].

[47] S.D. Badger, Direct Extraction Of One Loop Rational Terms, JHEP 01 (2009) 049 [arXiv:0806.4600] [SPIRES].

[48] Z. Bern, L.J. Dixon and D.A. Kosower, Bootstrapping multi-parton loop amplitudes in QCD, Phys. Rev. D 73 (2006) 065013 [hep-ph/0507005] [SPIRES].

[49] C.F. Berger, Z. Bern, L.J. Dixon, D. Forde and D.A. Kosower, Bootstrapping one-loop QCD amplitudes with general helicities, Phys. Rev. D 74 (2006) 036009 [hep-ph/0604195] [SPIRES].

[50] L.F. Alday, J.M. Henn, J. Plefka and T. Schuster, Scattering into the fifth dimension of $N=4$ super Yang-Mills, JHEP 01 (2010) 077 [arXiv:0908.0684] [SPIRES].

[51] M.L. Mangano and S.J. Parke, Multi-Parton Amplitudes in Gauge Theories, Phys. Rept. 200 (1991) 301 [hep-th/0509223] [SPIRES].

[52] Z. Bern, L.J. Dixon and D.A. Kosower, One-loop amplitudes for $e^{+} e^{-}$to four partons, Nucl. Phys. B 513 (1998) 3 [hep-ph/9708239] [SPIRES].

[53] M. Dinsdale, M. Ternick and S. Weinzierl, A comparison of efficient methods for the computation of Born gluon amplitudes, JHEP 03 (2006) 056 [hep-ph/0602204] [SPIRES].

[54] G. Georgiou, E.W.N. Glover and V.V. Khoze, Non-MHV Tree Amplitudes in Gauge Theory, JHEP 07 (2004) 048 [hep-th/0407027] [SPIRES].

[55] D. Binosi and L. Theussl, JaxoDraw: A graphical user interface for drawing Feynman diagrams, Comput. Phys. Commun. 161 (2004) 76 [hep-ph/0309015] [SPIRES].

[56] D. Binosi, J. Collins, C. Kaufhold and L. Theussl, JaxoDraw: A graphical user interface for drawing Feynman diagrams. Version 2.0 release notes, Comput. Phys. Commun. 180 (2009) 1709 [arXiv: 0811.4113] [SPIRES].

[57] J.A.M. Vermaseren, Axodraw, Comput. Phys. Commun. 83 (1994) 45 [SPIRES]. 
[58] D. Maitre and P. Mastrolia, S@M, a Mathematica Implementation of the Spinor-Helicity Formalism, Comput. Phys. Commun. 179 (2008) 501 [arXiv:0710.5559] [SPIRES].

[59] R.K. Ellis, W.T. Giele and G. Zanderighi, The one-loop amplitude for six-gluon scattering, JHEP 05 (2006) 027 [hep-ph/0602185] [SPIRES].

[60] J.L. Bourjaily, Efficient Tree-Amplitudes in $N=4$ : Automatic BCFW Recursion in Mathematica, arXiv:1011.2447 [SPIRES].

[61] N. Arkani-Hamed, F. Cachazo, C. Cheung and J. Kaplan, The S-matrix in Twistor Space, JHEP 03 (2010) 110 [arXiv:0903.2110] [SPIRES].

[62] J.L. Bourjaily, J. Trnka, A. Volovich and C. Wen, The Grassmannian and the Twistor String: Connecting All Trees in $N=4$ SYM, arXiv:1006.1899 [SPIRES].

[63] A. Hodges, Eliminating spurious poles from gauge-theoretic amplitudes, arXiv:0905.1473 [SPIRES].

[64] L. Mason and D. Skinner, Dual Superconformal Invariance, Momentum Twistors and Grassmannians, JHEP 11 (2009) 045 [arXiv:0909.0250] [SPIRES].

[65] Z. Bern, H. Ita and K. Ozeren, private communication.

[66] P. Uwer and B. Biedermann, private communication. 